\title{
ON THE DENSITY FUNCTIONS OF INTEGRALS OF GAUSSIAN RANDOM FIELDS
}

\author{
JINGCHEN LIU *** AND \\ GONGJUN XU, ${ }^{*}$ Columbia University
}

\begin{abstract}
In the paper we consider the density functions of random variables that can be written as integrals of exponential functions of Gaussian random fields. In particular, we provide closed-form asymptotic bounds for the density functions and, under smoothness conditions, we derive exact tail approximations of the density functions.
\end{abstract}

Keywords: Gaussian random field; integral; density function

2010 Mathematics Subject Classification: Primary 60F10; 60G70

\section{Introduction}

Consider a Gaussian random field $f(t)$ living on a $d$-dimensional compact set $T$. We say that $f(t)$ is a Gaussian random field if, for any finite subset $\left\{t_{1}, \ldots, t_{n}\right\} \subset T,\left(f\left(t_{1}\right), \ldots, f\left(t_{n}\right)\right)$ follows a multivariate Gaussian distribution. In this paper we consider the random variable

$$
\log \left(\int_{T} \mathrm{e}^{\sigma(t) f(t)} \mathrm{d} \vartheta(t)\right)
$$

for some positive function $\sigma(t)$ and a finite measure $\vartheta$. Of interest is the tail behavior of the density function of (1).

The integral of exponential functions of Gaussian random fields plays an important role in both applied probability and statistics, such as spatial point process, portfolio risk analysis, and option pricing (see [8] and [9]). As we focus on the theoretical development in this paper, we do not elaborate the applications, a detailed discussion of which can be found in Section 2 of [14].

The literature of extreme behavior of Gaussian random fields focuses mostly on the tail probabilities of $\sup _{T} f(t)$. The results contain general bounds as well as sharp asymptotic approximations as $b \rightarrow \infty$. A general upper bound for the tail of sup $f(x)$ is developed in [7] and [21], which is known as the Borel-TIS lemma. Several methods have been introduced to obtain bounds and asymptotic approximations (see [4], [16], [18], and [19]), each of which imposes different regularity conditions on the random fields. The density functions of sup $f(t)$ have also been studied in [6] and [20].

The distribution of the random variable in (1) is studied in the literature when $f(t)$ is a Brownian motion (see [10] and [22]). The tail approximation of the finite sum of correlated log-normal random variables has been studied in [3]. The corresponding simulation is studied in [5]. There are other general studies on heavy-tailed random variables that include the sum of log-normal random variables as special cases (see [11] and [15]). Recently, Liu [13] derived

Received 31 January 2012; revision received 24 September 2012.

* Postal address: Department of Statistics, Columbia University, 1255 Amsterdam Avenue, New York, NY 10027, USA.

** Email address: jcliu@ @stat.columbia.edu 
the asymptotic approximations of $\mathbb{P}\left(\int_{T} \mathrm{e}^{f(t)} \mathrm{d} t>b\right)$ as $b \rightarrow \infty$ for three times differentiable and homogeneous Gaussian random fields. Liu and $\mathrm{Xu}$ [14] further extended the results to the case when the process has a varying mean function.

The density function of (1) for a general Gaussian random field is still unexplored, which is the main target of this paper. The results derived in this paper lead immediately to bounds and approximations of the tail probabilities $\mathbb{P}\left(\int_{T} \mathrm{e}^{\sigma(t) f(t)} \mathrm{d} \vartheta(t)>b\right)$ by integrating the density on $[b, \infty)$. In addition, such a kind of local results provides technical support for the theoretical analysis of simulation studies, in which one typically needs to simulate a discrete process to approximate the continuous process. As shown in the technical development in [2] (focusing on the simulation of the tail probabilities of $\sup _{T} f(t)$ ), to provide bounds on the bias caused by the discretization, one needs local results (bounds of the density functions) of $\sup _{T} f(t)$.

The contribution of this paper is to develop asymptotic bounds and approximations of the density functions of (1). Our results consist of several theorems. Asymptotic upper bounds are given in Theorems 1 and 2 under different conditions. An exact approximation of the density is given in Theorem 3 when $f(t)$ is three times differentiable. In addition, during the proof of the theorems, a bound of $F^{\prime}(a)$ for all $a \in \mathbb{R}$ is derived (the results in Section 3.1.3).

The basic technique is to use the Karhunen-Loève expansion $f(t)=\sum_{i=1}^{\infty} x_{i} \phi_{i}(t)$ by developing bounds for $f_{N}(t)=\sum_{i=1}^{N} x_{i} \phi_{i}(t)$ and sending $N$ to $\infty$. For $f_{N}(t)$, we consider it to be a function of $\left(x_{1}, \ldots, x_{N}\right)$ and develop bounds of the integral on the surface $\left\{\left(x_{1}, \ldots, x_{N}\right): \log \int \mathrm{e}^{\sigma(t) f_{N}(t)} \mathrm{d} \varphi(t)=a\right\}$ (endowed with a standard Gaussian measure). Part of the analysis technique is inspired by Tsirel'son [20], who presented a bound of the density of $\sup _{T} f(t)$. The current analysis is more complicated in that $H_{f}$ is not a sublinear function of $f$, which is a crucial condition in the proof of [20]. Sun [18] also used this representation to derive an approximation of the tail probability of $\sup _{T} f(t)$. In addition, a change-of-measure technique is used to derive explicit forms of the bounds and the asymptotic approximations.

The organization of the rest of this paper is as follows. In Section 2 we present the main results. Proofs of the theorems are given in Section 3. Technical proofs of several lemmas are provided as supplemental material.

\section{Main results}

Consider a Gaussian random field, $f(t)$, living on a $d$-dimensional compact domain $T \subset \mathbb{R}^{d}$. For a finite measure $\vartheta$ on $T$ and a function $\sigma(t) \in(0, \infty)$ satisfying $\sigma_{T}=\sup _{t \in T} \sigma(t)<\infty$, let

$$
H_{f}:=\log \left(\int_{T} \mathrm{e}^{\sigma(t) f(t)} \mathrm{d} \vartheta(t)\right), \quad F(a):=\mathbb{P}\left(H_{f} \leq a\right) .
$$

Of interest is the probability density function $F^{\prime}(a)$. To facilitate the discussion, we present a list of conditions that we will refer to in later discussions.

(C1) The index domain $T$ is a $d$-dimensional compact subset of $\mathbb{R}^{d}$. The boundary $\partial T=$ $T-T^{o}$ is a piecewise smooth manifold, where $T^{o}$ is the interior of $T$. The measure $\vartheta$ is positive and $\vartheta(T)=1$.

(C2) The process $f(t)$ is almost surely continuous with zero mean and unit variance.

Furthermore, we impose two types of structures on the covariance function, under each of which we derive more precise bounds or approximations of $F^{\prime}(a)$.

(C3) The variance is constant, i.e. $\sigma(t) \equiv \sigma$. The measure $\vartheta$ has a positive and continuous density function with respect to the Lebesgue measure. The process $f(t)$ is homogeneous. 
The covariance function is $C(t)=\mathbb{E}(f(s) f(s+t))$, which satisfies the following two conditions.

(C3a) $C(t)$ satisfies the expansion

$$
C(t)=C(0)-|t|^{\alpha}+o\left(|t|^{\alpha}\right) \quad \text { as } t \rightarrow 0 \text { for } \alpha \in(0,2] .
$$

(C3b) For each $t \in \mathbb{R}^{d}, C(\lambda t)$ is a monotone decreasing function of $\lambda \in \mathbb{R}^{+}$.

(C4) The process $f(t)$ is almost surely at least three times continuously differentiable with respect to $t$. The Hessian matrix of $C(t)$ at the origin is $-I$, where $I$ is the $d \times d$ identity matrix.

Theorem 1. Suppose that conditions (C1) and (C2) are satisfied. Then, $F^{\prime}(a)$ exists almost everywhere and

$$
\limsup _{a \rightarrow \infty} \sigma_{T}^{2} a^{-1} \mathrm{e}^{a^{2} / 2 \sigma_{T}^{2}} F^{\prime}(a) \leq 1,
$$

where $\sigma_{T}=\sup _{t \in T} \sigma(t)<\infty$.

Remark 1. Under conditions (C1) and (C2) (very weak conditions), Theorem 1 establishes the existence and an asymptotic bound of $F^{\prime}(a)$. It also includes the case when $\vartheta$ is a discrete measure and, therefore, this bound is applicable to the finite/infinite sum of lognormal random variables. The following simple example implies that, without additional assumptions, the bound in Theorem 1 is efficient up to a polynomial term of $a$. Consider a constant field $f(t) \equiv Z$, where $Z \sim N(0,1)$. Let $\sigma(t)$ take a constant value $\sigma$. Then $F^{\prime}(a)=\exp \left(-a^{2} /\left(2 \sigma^{2}\right)\right) /(\sqrt{2 \pi} \sigma)$.

Under more regularity conditions, we further improve the bound.

Theorem 2. Suppose that conditions $(C 1)-(C 3)$ are satisfied. According to $(C 3)$, there exists some continuous function $\mu_{f}(t)$ on $T$ such that

$$
\mathrm{d} \vartheta(t)=\frac{\mathrm{e}^{\mu_{f}(t)}}{\int_{T} \mathrm{e}^{\mu_{f}(s)} \mathrm{d} s} \mathrm{~d} t .
$$

For each $\varepsilon$ and $a$, let $u_{\varepsilon}$ (as a function of $a$ ) be the solution to the equation

$$
\mathrm{e}^{\sigma u_{\varepsilon}} u_{\varepsilon}^{d \varepsilon-d / 2 \alpha}=\mathrm{e}^{a} \int_{T} \mathrm{e}^{\mu_{f}(t)} \mathrm{d} t .
$$

Then, for any $\varepsilon \in(0,1 /(2 \alpha))$,

$$
u_{\varepsilon}^{d \varepsilon-d / 2 \alpha-1} \mathrm{e}^{u_{\varepsilon}^{2} / 2} F^{\prime}(a) \rightarrow 0 \text { as } a \rightarrow \infty .
$$

Remark 2. Note that, when $a$ is large, (3) generally has two solutions. One is on the order of $a / \sigma$; the other one is close to 0 . We choose the larger solution as our $u_{\varepsilon}$.

In (3), if we replace the integral $\int \mathrm{e}^{\mu_{f}(t)} \mathrm{d} t$ by 1 (or any other constant) then $u_{\varepsilon}$ will be shifted by approximately a constant. Denote the corresponding solution by $\tilde{u}_{\varepsilon}$. Note that the results hold for all sufficiently small $\varepsilon$. For large enough $u_{\varepsilon}$, we have $\tilde{u}_{\varepsilon}<u_{\varepsilon / 2}$. Thus, the bound in (4) holds by replacing $u_{\varepsilon}$ with $\tilde{u}_{\varepsilon}$. 
The exact asymptotic approximation of $F^{\prime}(a)$ can be derived when $f$ is homogeneous and three times differentiable (condition (C4)). The statement of the theorem needs the following notation. Let ' $\partial$ ' denote the gradient and ' $\Delta$ ' denote the Hessian matrix with respect to $t$. The notation ' $\partial^{2}$ ' is used to denote the vector of second derivatives with respect to $t$, i.e. $\partial^{2} f(t)$ is a $d(d+1) / 2$-dimensional vector. The difference between $\partial^{2} f(t)$ and $\Delta f(t)$ is that $\Delta f(t)$ is a $d \times d$ symmetric matrix whose diagonal and upper triangle consist of elements of $\partial^{2} f(t)$.

It is well known that, for each given $t \in T,\left(f(t), \partial^{2} f(t)\right)$ is a multivariate Gaussian random vector with mean zero and covariance matrix

$$
\Gamma=\left(\begin{array}{cc}
1 & \mu_{20} \\
\mu_{02} & \mu_{22}
\end{array}\right)
$$

where $\mu_{20}$ is the vector containing the spectral moments of order two and $\mu_{22}$ is the matrix containing the spectral moments of order four. Both $\mu_{20}$ and $\mu_{22}$ are arranged in an appropriate order according to the order of $\partial^{2} f(t)$. See, e.g. the standard textbook [1, Chapter 5.5] for more details of $\mu_{20}$ and $\mu_{22}$.

Theorem 3. Suppose that conditions $(\mathrm{C1})-(\mathrm{C} 4)$ are satisfied (with the expansion in $(\mathrm{C} 3 a)$ replaced by $(C 4))$. Let $\vartheta$ be defined as in (2), and let $\mu_{f}(t)$ be three times differentiable. Then the following approximation holds as $a \rightarrow \infty$ :

$$
F^{\prime}(a)=(1+o(1)) \sigma^{-1} \tilde{u}^{d} \int_{T} \exp \left(-\frac{\left(\tilde{u}-\mu_{f}(t) / \sigma\right)^{2}}{2}\right) C_{H}\left(\mu_{f}, \sigma, t\right) \mathrm{d} t .
$$

Here $\tilde{u}$ (as a function of a) is the solution to

$$
\left(\frac{2 \pi}{\sigma}\right)^{d / 2} \tilde{u}^{-d / 2} \mathrm{e}^{\sigma \tilde{u}}=\mathrm{e}^{a} \int_{T} \mathrm{e}^{\mu_{f}(t)} \mathrm{d} t
$$

the function $C_{H}$ is defined as

$$
\begin{aligned}
C_{H}\left(\mu_{f}, \sigma, t\right)= & \frac{|\Gamma|^{-1 / 2}}{(2 \pi)^{(d+1)(d+2) / 4}} \\
& \times \exp \left(\frac{\mathbf{1}^{\top} \mu_{22} \mathbf{1}+\sum_{i} \partial_{i i i i}^{4} C(0)}{8 \sigma^{2}}+\frac{d \mu_{f}(t)+\operatorname{Tr}\left(\Delta \mu_{f}(t)\right)}{2 \sigma^{2}}+\frac{\left|\partial \mu_{f}(t)\right|^{2}}{\sigma^{2}}\right) \\
& \times \int_{z \in \mathbb{R}^{d(d+1) / 2}} \exp \left(-\frac{1}{2}\left[\frac{\left|\mu_{20} \mu_{22}^{-1} z\right|^{2}}{1-\mu_{20} \mu_{22}^{-1} \mu_{02}}+\left|\mu_{22}^{-1 / 2} z-\frac{\mu_{22}^{1 / 2} \mathbf{1}}{2 \sigma}\right|^{2}\right]\right) \mathrm{d} z,
\end{aligned}
$$

$\mu_{20}, \mu_{02}, \mu_{22}$, and $\Gamma$ are defined in (5), and

$$
\mathbf{1}=(\underbrace{1, \ldots, 1}_{d}, \underbrace{0, \ldots, 0}_{d(d-1) / 2})^{\top} .
$$

Remark 3. For condition (C1), if $\vartheta(T) \neq 1$, we can always perform the transformation

$$
\log \int_{T} \mathrm{e}^{\sigma f(t)} \mathrm{d} \vartheta(t)=\log \left(\frac{1}{\vartheta(T)} \int_{T} \mathrm{e}^{\sigma(t) f(t)} \mathrm{d} \vartheta(t)\right)+\log \vartheta(T)
$$

and let $\vartheta^{\prime}(\cdot)=\vartheta(\cdot) / \vartheta(T)$. 
Condition (C2) assumes the zero expectation function. For any continuous function $\mu(t)$,

$$
\log \int_{T} \mathrm{e}^{\sigma(t) f(t)+\mu(t)} \mathrm{d} \vartheta(t)=\log \int_{T} \mathrm{e}^{\sigma(t) f(t)} \mathrm{d} \vartheta^{\prime}(t),
$$

where $\mathrm{d} \vartheta^{\prime}(t)=\mathrm{e}^{\mu(t)} \mathrm{d} \vartheta(t)$. Therefore, this problem setting includes the situation when the mean is not a constant.

Condition $(\mathrm{C} 3 \mathrm{~b})$ is imposed for technical reasons to localize the integration around the maximum $t^{*}=\arg \sup _{t \in T} f(t)$. In particular, under condition $(\mathrm{C} 3 \mathrm{~b})$, the integral on the region $\left|t-t^{*}\right|>\varepsilon$ can be ignored in the analysis when $f\left(t^{*}\right)$ achieves a very high value. Condition (C3) includes quite a large class of homogeneous processes. One of the widely employed families is

$$
C(t)=\mathrm{e}^{-|t|^{\alpha}}
$$

for some $\alpha \in(0,2]$. Another more concrete example is given by

$$
f(t)=Z_{1} \cos (t)+Z_{2} \sin (t)
$$

for $t \in[0,2 \pi]$, where $Z_{1}$ and $Z_{2}$ are independent standard Gaussian random variables.

Condition (C4) implies that $C(t)$ is at least six times differentiable and the first, third, and fifth derivatives at the origin are all 0 . For the above exponential covariance functions, $(\mathrm{C} 4)$ is satisfied when $\alpha=2$. The assumption that the Hessian matrix is the identity is introduced to simplify the notation. For any Gaussian process $g(t)$ with covariance function $C_{g}(t)$ and $\Delta C_{g}(0)=-\Sigma$ and $\operatorname{det}(\Sigma)>0$, this assumption can be obtained by an affine transformation by letting $g(t)=f\left(\Sigma^{1 / 2} t\right)$ and

$$
\log \int_{T} \mathrm{e}^{\sigma g(t)+\mu_{f}(t)} \mathrm{d} t=\log \operatorname{det}\left(\Sigma^{-1 / 2}\right)+\log \int_{\left\{s: \Sigma^{-1 / 2} s \in T\right\}} \mathrm{e}^{\sigma f(s)+\mu_{f}\left(\Sigma^{-1 / 2} s\right)} \mathrm{d} s,
$$

where, for each positive semidefinite matrix $\Sigma$, we let $\Sigma^{1 / 2}$ be a symmetric matrix such that $\Sigma^{1 / 2} \Sigma^{1 / 2}=\Sigma$.

\section{Proofs}

In this section we present the proofs of the theorems. We organize the proofs as follows. In Section 3.1 we develop a proposition that is central to the proofs of all the three theorems. The theorems are proved in Section 3.2 based on the results in Section 3.1. To streamline the discussion, we present the statements of lemmas where they are applied and postpone their proofs to Appendix A.

Throughout the discussion, we use the following notation for the asymptotic behaviors. We say that $0 \leq g(a)=O(h(a))$ if $g(a) \leq \operatorname{ch}(a)$ for some constant $c \in(0, \infty)$ and all $a \geq a_{0}>0$; similarly, $g(a)=o(h(a))$ if $g(a) / h(a) \rightarrow 0$ as $a \rightarrow \infty$.

\subsection{A general bound for $F^{\prime}(a)$}

Proposition 1. Under the conditions of Theorem 1, $F^{\prime}(a)$ exists almost everywhere. Choose $b<a$ (depending on $a$ ) such that $a-b \rightarrow 0$ and $a(a-b) \rightarrow \infty$ when we send $a$ to $\infty$. Then

$$
\limsup _{a \rightarrow \infty} \sqrt{2 \pi} \sigma_{T} \exp \left(\frac{\sigma_{T}^{2} t_{b}^{2}+2(a-b) b}{2 \sigma_{T}^{2}}\right) F^{\prime}(a) \leq 1,
$$

where $\sigma_{T}=\sup _{t \in T} \sigma(t), t_{b}=\Phi^{-1}(F(b))$, and $\Phi(\cdot)$ is the cumulative distribution function of the standard Gaussian distribution. 
We dedicate the rest of this subsection to proving this proposition. According to the Karhunen-Loève representation theorem (see Chapter 3 of [1]), $f(t)$ has the expression

$$
f(t)=\sum_{i=1}^{\infty} x_{i} \phi_{i}(t)
$$

where $\left\{x_{i}, i \in \mathbb{N}\right\}$ are independent and identically distributed standard Gaussian random variables and $\sum_{i} \phi_{i}(t)^{2}=1$. For any positive integer $N$, let $f_{N}(t)$ be the partial sum of the first $N$ terms. Note that $f_{N}(t)$ can be viewed as a function of $\left(x_{1}, \ldots, x_{N}\right)$. We slightly abuse the notation and write

$$
f_{N}(x, t)=\sum_{i=1}^{N} x_{i} \phi_{i}(t)
$$

where $x:=\left(x_{1}, \ldots, x_{N}\right)$. When writing $f_{N}(t)$, we consider it to be a random function; when writing $f_{N}(x, t)$ or $f_{N}(x, \cdot)$ we emphasize that it is a function of $x$ mapping from $\mathbb{R}^{N}$ to $C(T)$. Similarly, we redefine function $H_{f_{N}}: \mathbb{R}^{N} \rightarrow \mathbb{R}$ as

$$
H_{f_{N}}(\cdot): x \mapsto H_{f_{N}}(x)=\log \left(\int_{T} \mathrm{e}^{\sigma(t) f_{N}(x, t)} \mathrm{d} \vartheta(t)\right) .
$$

Let $\mu_{N}$ be the standard Gaussian measure on the probability space $\left(\mathbb{R}^{N}, \mathcal{B}\left(\mathbb{R}^{N}\right), \mu_{N}\right)$ with density function

$$
\varphi_{N}(x)=(2 \pi)^{-N / 2} \exp \left(-\frac{1}{2}|x|^{2}\right),
$$

that is, $\mu_{N}(A)=\int_{A} \varphi_{N}(x) \mathrm{d} x$, where $|\cdot|$ is the Euclidean distance.

We first establish a bound for the density of $f_{N}(t)$ and then send $N$ to $\infty$. On the probability space $\left(\mathbb{R}^{N}, \mathscr{B}\left(\mathbb{R}^{N}\right), \mu_{N}\right)$, we define the sets

$$
V_{N, a}:=\left\{x \in \mathbb{R}^{N}: H_{f_{N}}(x) \leq a\right\}, \quad W_{N, a}:=\left\{x \in \mathbb{R}^{N}: \sup _{t \in T}\left\{\sigma(t) f_{N}(x, t)\right\} \leq a\right\},
$$

and the distribution functions

$$
\begin{gathered}
F_{N}(a):=\mathbb{P}\left(H_{f_{N}} \leq a\right)=\mu_{N}\left(V_{N, a}\right), \\
G_{N}(a):=\mathbb{P}\left(\sup _{t \in T}\left\{\sigma(t) f_{N}(t)\right\} \leq a\right)=\mu_{N}\left(W_{N, a}\right) .
\end{gathered}
$$

We prove Proposition 1 in four steps. In steps 1 and 2, we derive a 'not-so-friendly' bound for $F_{N}^{\prime}(a)$. In step 3 we send $N$ to $\infty$ and develop the corresponding bound for $F^{\prime}(a)$. Finally, inequality (6) is proved in step 4 based on the results in step 3.

3.1.1. Step 1. Let $\nabla H_{f_{N}}(x)$ be the gradient field of $H_{f_{N}}(x)$ with respect to $x$, and define

$$
l_{x}=\frac{1}{\left|\nabla H_{f_{N}}(x)\right|} .
$$

Furthermore, let $S_{a}$ be the surface on which $H_{f_{N}}(x)=a$, i.e.

$$
S_{a}=\left\{x: H_{f_{N}}(x)=a\right\} .
$$

We write

$$
\tilde{f}_{N}(x, t)=\sigma(t) f_{N}(x, t)
$$


For $a \in \mathbb{R}$, the density function $F_{N}^{\prime}(a)$ can be written as a surface integral as follows:

$$
\begin{aligned}
F_{N}^{\prime}(a) & =\lim _{\varepsilon \rightarrow 0} \frac{F_{N}(a+\varepsilon)-F_{N}(a)}{\varepsilon} \\
& =\lim _{\varepsilon \rightarrow 0} \frac{\mu_{N}\left(V_{a+\varepsilon}\right)-\mu_{N}\left(V_{a}\right)}{\varepsilon} \\
& =\int_{S_{a}} l_{x} \varphi_{N}(x) \mathrm{d} S_{a}(x) .
\end{aligned}
$$

Here $\varphi_{N}$ is defined as in (7) and $\mathrm{d} S_{a}(x)$ denotes the surface integral element on $S_{a} \subset \mathbb{R}^{N}$.

The next lemma gives a basic inequality that bounds the surface integral by an integral on the set $V_{N, a}$. Its proof follows a similar derivation as in [20].

Lemma 1. Consider the probability space $\left(\mathbb{R}^{N}, \mathcal{B}\left(\mathbb{R}^{N}\right), \mu_{N}\right)$. Under the conditions in Theorem 1, we have the bound

$$
\int_{S_{a}} l_{x} \varphi_{N}(x) \mathrm{d} S_{a}(x) \leq \int_{V_{N, a}^{c}} l_{h(x)}\left(c_{h(x)}^{+}+1\right) \mathrm{d} \mu_{N}(x),
$$

where $V_{N, a}^{c}=\mathbb{R}^{N} \backslash V_{N, a}, h(x)=\arg \min _{z \in S_{a}}|x-z|$ is the projection of $x$ onto the surface $S_{a}$,

$$
c_{x}=\left\langle x, \boldsymbol{n}_{x}\right\rangle, \quad c_{x}^{+}=\max \left\{c_{x}, 0\right\},
$$

$\langle\cdot, \cdot\rangle$ is the inner product, and $\boldsymbol{n}_{x}$ is the unit vector orthogonal to the surface $S_{a}$ pointing towards the side where $H_{f_{N}}(x)$ has larger values.

3.1.2. Step 2. We start with deriving bounds for $l_{h(x)}$ and $c_{h(x)}$, where $h(x)$ is defined as in Lemma 1. Note that

$$
\partial_{i} H_{f_{N}}(h(x))=\mathrm{e}^{-a} \int_{T} \sigma(t) \phi_{i}(t) \mathrm{e}^{\tilde{f}_{N}(h(x), t)} \mathrm{d} \vartheta(t)
$$

and, since $h(x) \in S_{a}$,

$$
\begin{aligned}
l_{h(x)}^{-1} c_{h(x)} & =\left\langle h(x), \nabla H_{f_{N}}(h(x))\right\rangle \\
& =\mathrm{e}^{-a} \int_{T} \tilde{f}_{N}(h(x), t) \mathrm{e}^{\tilde{f}_{N}(h(x), t)} \mathrm{d} \vartheta(t) \\
& \leq \sup _{t \in T}\left\{\tilde{f}_{N}(h(x), t)\right\} \mathrm{e}^{-a} \int_{T} \mathrm{e}^{\tilde{f}_{N}(h(x), t)} \mathrm{d} \vartheta(t) \\
& =\sup _{t \in T}\left\{\tilde{f}_{N}(h(x), t)\right\} .
\end{aligned}
$$

This implies that

$$
c_{h(x)}^{+} \leq l_{h(x)}\left(\sup _{t \in T}\left\{\tilde{f}_{N}(h(x), t)\right\}\right)^{+} .
$$

The following two lemmas provide a bound for $l_{h(x)}$.

Lemma 2. $V_{N, a}$ is a convex set and $H_{f_{N}}: \mathbb{R}^{N} \rightarrow \mathbb{R}$ is a convex function. 
Lemma 3. For each $b<a$ and $x \in S_{a}$,

$$
l_{x}=\left|\nabla H_{f_{N}}(x)\right|^{-1} \leq \frac{\rho\left(x, V_{N, b}\right)}{a-b},
$$

where $V_{N, b}=\left\{z \in \mathbb{R}^{N}: H_{f_{N}}(z) \leq b\right\}$ and $\rho\left(x, V_{N, b}\right)=\inf _{z \in V_{N, b}}|x-z|$.

According to (12) and Lemma 3, for each $x \in V_{N, a}^{c}$, the integrant in (10) is bounded by

$$
l_{h(x)}\left(c_{h(x)}^{+}+1\right) \leq \frac{\rho\left(h(x), V_{N, b}\right)}{a-b}\left(\frac{\rho\left(h(x), V_{N, b}\right)}{a-b}\left(\sup _{t \in T}\left\{\tilde{f}_{N}(h(x), t)\right\}\right)^{+}+1\right),
$$

which implies that

$$
\begin{aligned}
F_{N}^{\prime}(a) & \leq \int_{V_{N, a}^{c}} l_{h(x)}\left(c_{h(x)}^{+}+1\right) \mathrm{d} \mu_{N}(x) \\
& \leq \int_{V_{N, a}^{c}} \frac{\rho\left(h(x), V_{N, b}\right)}{a-b}\left(\frac{\rho\left(h(x), V_{N, b}\right)}{a-b}\left(\sup _{t \in T}\left\{\tilde{f}_{N}(h(x), t)\right\}\right)^{+}+1\right) \mathrm{d} \mu_{N}(x) .
\end{aligned}
$$

By the fact that, for any $x$,

$$
\log \left(\int_{t \in T} \mathrm{e}^{\tilde{f}_{N}(x, t)} \mathrm{d} \vartheta(t)\right) \leq \sup _{t \in T}\left\{\tilde{f}_{N}(x, t)\right\},
$$

we obtain $W_{N, a} \subseteq V_{N, a}$ for all $a$, where $W_{N, a}$ is defined as in (8). Now, for some constant $M \geq 1$, partition $V_{N, a}^{c}=\mathbb{R}^{N} \backslash V_{N, a}$ into two parts:

$$
V_{N, a}^{c}=V_{N, a, 1}^{c} \cup V_{N, a, 2}^{c},
$$

where

$$
V_{N, a, 1}^{c}=\left\{x+\lambda \boldsymbol{n}_{x}: \lambda \geq 0, x \in S_{a}, \text { and } \sup _{t \in T}\left\{\tilde{f}_{N}(x, t)\right\}<M a\right\}
$$

and

$$
V_{N, a, 2}^{c}=\left\{x+\lambda \boldsymbol{n}_{x}: \lambda \geq 0, x \in S_{a}, \text { and } \sup _{t \in T}\left\{\tilde{f}_{N}(x, t)\right\} \geq M a\right\}
$$

with $\boldsymbol{n}_{x}$ defined as in Lemma 1. Figure 1 illustrates the relative geometric positions of all the relevant sets.

We split the integral in (13) into two parts:

$$
\begin{aligned}
F_{N}^{\prime}(x) \leq & \int_{V_{N, a, 1}^{c}} \frac{\rho\left(h(x), V_{N, b}\right)}{a-b}\left(\frac{\rho\left(h(x), V_{N, b}\right)}{a-b}\left(\sup _{t \in T}\left\{\tilde{f}_{N}(h(x), t)\right\}\right)^{+}+1\right) \mathrm{d} \mu_{N}(x) \\
& +\int_{V_{N, a, 2}^{c}} \frac{\rho\left(h(x), V_{N, b}\right)}{a-b}\left(\frac{\rho\left(h(x), V_{N, b}\right)}{a-b}\left(\sup _{t \in T}\left\{\tilde{f}_{N}(h(x), t)\right\}\right)^{+}+1\right) \mathrm{d} \mu_{N}(x) \\
= & I_{1}+I_{2} .
\end{aligned}
$$

We consider the integrals $I_{1}$ and $I_{2}$ separately. When $G_{N}(a)=\mathbb{P}\left(\sup _{t \in T}\left\{\tilde{f}_{N}(t)\right\} \leq a\right) \leq \frac{1}{2}$, we take $M=1$. Note that in this case, by the fact that $W_{N, a} \subseteq V_{N, a}$, the first term on the right-hand side of the above display vanishes and we only need to consider the second integral. Then, for 


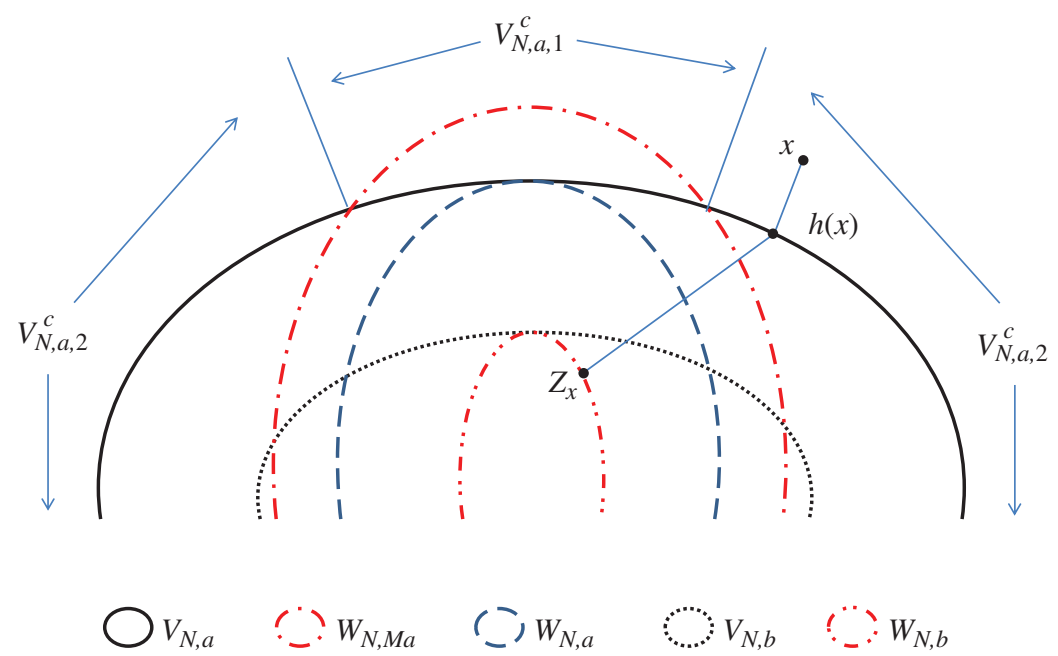

Figure 1: An illustration of the relative positions of different sets used in the proof. The legends indicate the boundary of each set.

the first integral, we only consider the case in which $G_{N}(a)>\frac{1}{2}$ (note that $G_{N}(a)>\frac{1}{2}$ implies that $a>0$ ).

A bound for $I_{1}$. By the definition of $V_{N, a, 1}^{c}, x \in V_{N, a, 1}^{c}$ implies that $\sup _{t \in T}\left\{\tilde{f}_{N}(h(x), t)\right\}<$ $M a$ and, therefore, we have, for $a>0$,

$$
I_{1} \leq \int_{V_{N, a, 1}^{c}} \frac{\rho\left(h(x), V_{N, b}\right)}{a-b}\left(\frac{\rho\left(h(x), V_{N, b}\right)}{a-b} M a+1\right) \mathrm{d} \mu_{N}(x) .
$$

The following lemma provides a bound for $I_{1}$.

Lemma 4. For any $a>b$ with $G_{N}(b)>\frac{1}{2}$ and increasing function $J(\cdot)$, we have

$$
\int_{V_{N, a, 1}^{c}} J\left(\rho\left(x, V_{N, b}\right)\right) \mathrm{d} \mu_{N}(x) \leq \int_{\tau_{M, a, b}+t_{N, b}}^{\infty} J\left(u-t_{N, b}\right) \mathrm{d} \Phi(u),
$$

where

$$
t_{N, b}=\Phi^{-1}\left(\mu_{N}\left(V_{N, b}\right)\right), \quad \tau_{M, a, b}=\frac{a-b}{M a}\left(\frac{a-b}{M a}+1\right) t_{N, b}^{\prime}
$$

with

$$
t_{N, b}^{\prime}=\Phi^{-1}\left(\mathbb{P}\left(H_{\tilde{f}_{N}}<b, \sup _{t \in T}\left\{\tilde{f}_{N}(t)\right\}<M a\right)\right) .
$$

According to Lemma 4, for any $b<a$ such that $G_{N}(b)>\frac{1}{2}$, the right-hand side of (14) satisfies the inequality

$$
\begin{aligned}
\int_{V_{N, a, 1}^{c}} & \frac{\rho\left(h(x), V_{N, b}\right)}{a-b}\left(\frac{\rho\left(h(x), V_{N, b}\right)}{a-b} M a+1\right) \mathrm{d} \mu_{N}(x) \\
\leq & \int_{\tau_{M, a, b}+t_{N, b}}^{\infty} \frac{u-t_{N, b}}{a-b}\left(\frac{u-t_{N, b}}{a-b} M a+1\right) \mathrm{d} \Phi(u) .
\end{aligned}
$$

This integral can be further bounded by the following inequality, whose proof is given in [20]. 
Lemma 5. For the standard normal distribution, we have

$$
\int_{t+r}^{\infty}(u-t)^{k} \mathrm{~d} \Phi(u) \leq(1-\Phi(t+r)) r^{k} k ! \sum_{i=0}^{k} \frac{1}{(k-i) ! r^{i}(t+r)^{i}} \quad \text { for } k \in \mathbb{N}, r>0 .
$$

Applying Lemma 5 to (15) we obtain

$$
I_{1} \leq\left(1-\Phi\left(\tau_{M, a, b}+t_{N, b}\right)\right) C_{1}\left(a, b, \tau_{M, a, b}\right)
$$

where

$$
\begin{aligned}
C_{1}\left(a, b, \tau_{M, a, b}\right)= & \frac{M a}{(a-b)^{2}} \tau_{M, a, b}^{2}+\frac{\tau_{M, a, b}}{a-b}+\frac{2 M a \tau_{M, a, b}+a-b}{(a-b)^{2}\left(\tau_{M, a, b}+t_{N, b}\right)} \\
& +\frac{2 M a}{(a-b)^{2}\left(\tau_{M, a, b}+t_{N, b}\right)^{2}} .
\end{aligned}
$$

A bound for $I_{2}$. Choose another constant $\tilde{b}<a$. Given the fact that $W_{N, \tilde{b}} \subseteq V_{N, \tilde{b}}$, we have

$$
I_{2} \leq \int_{V_{N, a, 2}^{c}} \frac{\rho\left(x, W_{N, \tilde{b}}\right)}{a-\tilde{b}}\left(\frac{\rho\left(x, W_{N, \tilde{b}}\right)}{a-\tilde{b}}\left(\sup _{t \in T}\left\{\tilde{f}_{N}(h(x), t)\right\}\right)^{+}+1\right) \mathrm{d} \mu_{N}(x) .
$$

We use the following lemma to further bound $I_{2}$.

Lemma 6. Consider the probability space $\left(\mathbb{R}^{N}, \mathscr{B}\left(\mathbb{R}^{N}\right), \mu_{N}\right)$ and a positive measure set $B$. For any increasing function $J$ on $\mathbb{R}^{+}$and $r>0$, we have

$$
\int_{B_{r}^{c}} J(\rho(x, B)) \mathrm{d} \mu_{N}(x) \leq \int_{t_{B}+r}^{\infty} J\left(u-t_{B}\right) \mathrm{d} \Phi(u),
$$

where $B_{r}^{c}=\mathbb{R}^{N} \backslash B_{r}=\{x: \rho(x, B)>r\}$ and $t_{B}:=\Phi^{-1}\left(\mu_{N}(B)\right)$.

In order to use Lemma 6 with $B=W_{N, \tilde{b}}$, we need to derive a lower bound for $\rho\left(x, W_{N, \tilde{b}}\right)$ for $x \in V_{N, a, 2}^{c}$ (so that each $x \in V_{N, a, 2}^{c}$ is reasonably far away from $W_{N, \tilde{b}}$ and $V_{N, a, 2}^{c} \subseteq B_{r}^{c}$ ) and an upper bound for $\sup _{t \in T}\left\{\tilde{f}_{N}(h(x), t)\right\}, h(x) \in S_{a}$.

Let $\phi^{N}(t)=\left(\phi_{1}(t), \ldots, \phi_{N}(t)\right)$. For any $z \in W_{N, \tilde{b}}$, any unit-length vector $\boldsymbol{v}$ and scalar $\lambda$,

$$
\begin{aligned}
\sup _{t \in T}\left\{\tilde{f}_{N}(z+\lambda \boldsymbol{v}, t)\right\} & =\sup _{t \in T}\left\{\sigma(t)\left\langle z+\lambda \boldsymbol{v}, \phi^{N}(t)\right\rangle\right\} \\
& \leq \sup _{t \in T}\left\{\sigma(t)\left\langle z, \phi^{N}(t)\right\rangle\right\}+\sup _{t \in T}\left\{\sigma(t)\left\langle\lambda \boldsymbol{v}, \phi^{N}(t)\right\rangle\right\} \\
& \leq \tilde{b}+\lambda \sigma_{T} .
\end{aligned}
$$

Let $\lambda<(M a-\tilde{b}) / \sigma_{T}$. For any unit-length vector $\boldsymbol{v}$, we have

$$
\sup _{t \in T}\left\{\tilde{f}_{N}\left(z+\frac{M a-\tilde{b}}{\sigma_{T}} \boldsymbol{v}, t\right)\right\}<M a .
$$

Thus, for any point $x \in \mathbb{R}^{N}$, if $\rho\left(x, W_{N, \tilde{b}}\right)<(M a-\tilde{b}) / \sigma_{T}$ then $\sup _{t}\left\{\tilde{f}_{N}(x, t)\right\}<M a$. Therefore, for any $x \in V_{N, a, 2}^{c}$, we have $\rho\left(h(x), W_{N, \tilde{b}}\right) \geq(M a-\tilde{b}) / \sigma_{T}$. Given that $W_{N, \tilde{b}} \subseteq V_{N, a}$ 
and that $V_{N, a}$ is a convex set, we obtain $\left\langle h(x)-y, \boldsymbol{n}_{h(x)}\right\rangle>0$ for all $y \in W_{N, \tilde{b}}$. Thus, we obtain

$$
\rho\left(x, W_{N, \tilde{b}}\right) \geq \rho\left(h(x), W_{N, \tilde{b}}\right) \geq \frac{M a-\tilde{b}}{\sigma_{T}} .
$$

Thus, we have derived a lower bound of $\rho\left(x, W_{N, \tilde{b}}\right)$ for $x \in V_{N, a, 2}^{c}$. See Figure 1 for an illustration.

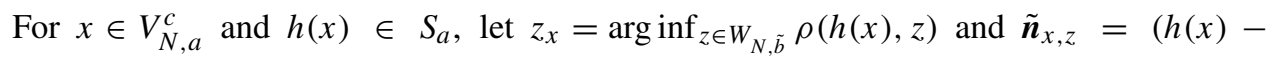
$\left.z_{x}\right) /\left|h(x)-z_{x}\right|$ (see Figure 1). An upper bound for $\sup _{t \in T}\left\{\tilde{f}_{N}(h(x), t)\right\}$ is

$$
\sup _{t \in T}\left\{\tilde{f}_{N}(h(x), t)\right\}=\sup _{t \in T}\left\{\tilde{f}_{N}\left(z_{x}+\rho\left(h(x), W_{\tilde{b}}\right) \tilde{\boldsymbol{n}}_{x_{z}}, t\right)\right\} \leq \rho\left(x, W_{\tilde{b}}\right) \sigma_{T}+\tilde{b},
$$

where the last step follows exactly the same argument as in (18). Thus, substituting the above bound for $\sup _{t \in T}\left\{\tilde{f}_{N}(h(x), t)\right\}$ into (17), we have

$$
\begin{aligned}
\int_{V_{N, a, 2}^{c}} & l_{h(x)}\left(c_{h(x)}^{+}+1\right) \mathrm{d} \mu_{N}(x) \\
\leq & \int_{V_{N, a, 2}^{c}}\left[\left(\frac{\rho\left(x, W_{N, \tilde{b}}\right)}{a-\tilde{b}}\right)^{2}\left(\rho\left(x, W_{N, \tilde{b}}\right) \sigma_{T}+\tilde{b}^{+}\right)+\frac{\rho\left(x, W_{N, \tilde{b}}\right)}{a-\tilde{b}}\right] \mathrm{d} \mu_{N}(x) .
\end{aligned}
$$

Then, by Lemma $6\left(B=W_{N, \tilde{b}}\right.$ and $\left.V_{N, a, 2}^{c} \subseteq B_{(M a-\tilde{b}) / \sigma_{T}}^{c}\right)$, the inequality

$$
\begin{aligned}
\int_{V_{N, a, 2}^{c}} & {\left[\left(\frac{\rho\left(x, W_{N, \tilde{b}}\right)}{a-\tilde{b}}\right)^{2}\left(\rho\left(x, W_{N, \tilde{b}}\right) \sigma_{T}+\tilde{b}^{+}\right)+\frac{\rho\left(x, W_{N, \tilde{b}}\right)}{a-\tilde{b}}\right] \mathrm{d} \mu_{N}(x) } \\
\leq & \int_{r_{M, a, \tilde{b}}+t_{W_{N, \tilde{b}}}}^{\infty}\left[\left(\frac{u-t_{W_{N, \tilde{b}}}}{a-\tilde{b}}\right)^{2}\left(\left(u-t_{W_{N, \tilde{b}}}\right) \sigma_{T}+\tilde{b}^{+}\right)+\frac{u-t_{W_{N, \tilde{b}}}}{a-\tilde{b}}\right] \mathrm{d} \Phi(u),
\end{aligned}
$$

holds, where

$$
t_{W_{N, \tilde{b}}}=\Phi^{-1}\left(G_{N}(\tilde{b})\right) \quad \text { and } \quad r_{M, a, \tilde{b}}=\frac{M a-\tilde{b}}{\sigma_{T}} .
$$

By Lemma 5, the above integral is bounded by

$$
\left(1-\Phi\left(r_{M, a, \tilde{b}}+t_{W_{N, \tilde{b}}}\right)\right) C_{2}\left(a, \tilde{b}, r_{M, a, \tilde{b}}\right),
$$

where

$$
\begin{aligned}
C_{2}\left(a, \tilde{b}, r_{M, a, \tilde{b}}\right):= & \frac{r_{M, a, \tilde{b}}}{a-\tilde{b}}+\frac{1}{(a-\tilde{b})\left(t_{W_{N, \tilde{b}}}+r_{M, a, \tilde{b}}\right)} \\
& +\frac{\tilde{b}^{+}}{(a-\tilde{b})^{2}} \sum_{i=0}^{2} \frac{r_{M, a, \tilde{b}}^{2} 2 !}{(2-i) ! r_{M, a, \tilde{b}}^{i}\left(t_{W_{N, \tilde{b}}}+r_{M, a, \tilde{b}}\right)^{i}} \\
& +\frac{\sigma_{T}}{(a-\tilde{b})^{2}} \sum_{i=0}^{3} \frac{r_{M, a, \tilde{b}}^{3} 3 !}{(3-i) ! r_{M, a, \tilde{b}}^{i}\left(t_{W_{N, \tilde{b}}}+r_{M, a, \tilde{b}}\right)^{i}} .
\end{aligned}
$$

Combining (16) and (19), we have, for $a$ such that $G_{N}(a)>G_{N}(b)>\frac{1}{2}$ and $\tilde{b}<a$,

$$
\begin{aligned}
F_{N}^{\prime}(a) \leq \min _{M \geq 1}\left\{\left(1-\Phi\left(\tau_{M, a, b}+t_{N, b}\right)\right) C_{1}\left(a, b, \tau_{M, a, b}\right)\right. \\
\left.+\left(1-\Phi\left(r_{M, a, \tilde{b}}+t_{W_{N, \tilde{b}}}\right)\right) C_{2}\left(a, \tilde{b}, r_{M, a, \tilde{b}}\right)\right\},
\end{aligned}
$$


and, for $a$ satisfying $G_{N}(a) \leq \frac{1}{2}$, by taking $M=1$ we have, for constant $\tilde{b}<a$,

$$
\begin{aligned}
F_{N}^{\prime}(a) & \leq \int_{V_{N, a, 2}^{c}} \frac{\rho\left(h(x), V_{N, \tilde{b}}\right)}{a-\tilde{b}}\left(\frac{\rho\left(h(x), V_{N, \tilde{b}}\right)}{a-\tilde{b}}\left(\sup _{t \in T}\left\{\tilde{f}_{N}(h(x), t)\right\}\right)^{+}+1\right) \mathrm{d} \mu_{N}(x) \\
& \leq\left(1-\Phi\left(r_{a, \tilde{b}}+t_{W_{N, \tilde{b}}}\right)\right) C_{2}\left(a, \tilde{b}, r_{a, \tilde{b}}\right),
\end{aligned}
$$

with $r_{a, \tilde{b}}=(a-\tilde{b}) / \sigma_{T}$.

3.1.3. Step 3: extension to $f(t)$. From the above derivations, the $F_{N}(a)$ are continuously differentiable on $\mathbb{R}$. Let

$$
D^{+} F_{N}^{\prime}(a):=\limsup _{\varepsilon \rightarrow 0} \frac{F_{N}^{\prime}(a+\varepsilon)-F_{N}^{\prime}(a)}{\varepsilon} .
$$

By Lemma 8 (presented in Appendix A), the total variation of $F_{N}^{\prime}$ on any interval $\left[a_{1}, a_{2}\right]$ satisfies

$$
\bigvee_{a_{1}}^{a_{2}} F_{N}^{\prime} \leq \sup _{a \in\left[a_{1}, a_{2}\right]} F_{N}^{\prime}(a)+2\left(a_{2}-a_{1}\right) \sup _{a \in\left[a_{1}, a_{2}\right]} D^{+} F_{N}^{\prime}(a) \leq m_{1}+m_{2}\left(a_{2}-a_{1}\right)
$$

for some constants $m_{1}, m_{2}>0$. Therefore, $F_{N}^{\prime}(a)$ is continuous on $\left[a_{1}, a_{2}\right]$ except for a countable set. Also, $F_{N}^{\prime}(a)$ is bounded in the $L^{1}$ norm on the interval $\left[a_{1}, a_{2}\right]$. Then, by Helly's selection theorem, there exists a subsequence $\left\{F_{N_{i}}^{\prime}\right\}_{i}$ such that it converges almost everywhere (and also in the $L^{1}$ norm) to a function $\tilde{F}^{\prime}$ of bounded total variation on $\left[a_{1}, a_{2}\right]$. Note that $F_{N}(a)$ converges uniformly to $F(a)$ on the interval $\left[a_{1}, a_{2}\right]$ (see Theorem 3.1.2 of [1]). Thus,

$$
F\left(a_{2}\right)-F\left(a_{1}\right)=\lim _{i} F_{N_{i}}\left(a_{2}\right)-F_{N_{i}}\left(a_{1}\right)=\lim _{i} \int_{a_{1}}^{a_{2}} F_{N_{i}}^{\prime}(b) \mathrm{d} b=\int_{a_{1}}^{a_{2}} \tilde{F}^{\prime}(b) \mathrm{d} b,
$$

which implies that $\tilde{F}^{\prime}=F^{\prime}$ almost everywhere on $\left[a_{1}, a_{2}\right]$. Therefore, by the convergence result, we obtain an upper bound of $F^{\prime}(a)$ by sending $N$ to $\infty$ on both sides of (21), i.e. for $G(a)>G(b)>\frac{1}{2}$ and $\tilde{b}<a\left(\right.$ where $\left.G(a)=\mathbb{P}\left(\sup _{t} \tilde{f}(t) \leq a\right)\right)$, we have

$$
\begin{aligned}
F^{\prime}(a) \leq \min _{M \geq 1}\left\{\left(1-\Phi\left(\tau_{M, a, b}+t_{b}\right)\right) C_{1}\left(a, b, \tau_{M, a, b}\right)\right. \\
\left.+\left(1-\Phi\left(r_{M, a, \tilde{b}}+t_{W_{\tilde{b}}}\right)\right) C_{2}\left(a, \tilde{b}, r_{M, a, \tilde{b}}\right)\right\},
\end{aligned}
$$

and, for $a$ such that $G(a) \leq \frac{1}{2}$ and $\tilde{b}<a$,

$$
F^{\prime}(a) \leq\left(1-\Phi\left(r_{a, \tilde{b}}+t_{W_{\tilde{b}}}\right)\right) C_{2}\left(a, \tilde{b}, r_{a, \tilde{b}}\right),
$$

where $t_{b}=\Phi^{-1}(F(b)), t_{W_{\tilde{b}}}=\Phi^{-1}(G(\tilde{b})), r_{M, a, \tilde{b}}=(M a-\tilde{b}) / \sigma_{T}, r_{a, \tilde{b}}=(a-\tilde{b}) / \sigma_{T}$, and, with a slight abuse of notation,

$$
\tau_{M, a, b}=\frac{a-b}{M a}\left(\frac{a-b}{M a}+1\right) t_{b}^{\prime} \quad \text { with } \quad t_{b}^{\prime}=\Phi^{-1}\left(\mathbb{P}\left(H_{f}<b, \sup _{t \in T} f(t)<M a\right)\right) .
$$


3.1.4. Step 4. Based on the result in (22), we now prove Proposition 1 in step 4.

By the Borel-TIS lemma (Lemma 9 in Appendix B; see also [7], [21], and Theorem 2.1.1 of [1]), we have

$$
\liminf _{a \rightarrow \infty} \frac{t_{a}}{a} \geq \lim _{a \rightarrow \infty} \frac{t_{W_{a}}}{a}=\sigma_{T}^{-1} \quad \text { and } \quad t_{W_{a}}-\sigma_{T}^{-1} a=O(1) .
$$

Now choose $b=b(a)<a$ such that, as $a \rightarrow \infty, a-b \rightarrow 0$ and $a(a-b) \rightarrow \infty$, and

$$
M=\sigma_{T}\left(1+\frac{C}{a}\right) \frac{t_{b}}{b}>1
$$

with a large enough constant $C$ (note that $t_{b} \geq t_{W_{b}} \geq b / \sigma_{T}+O(1)$ ). In addition, let $\tilde{b}$ be a fixed constant. Under the above settings, as $a \rightarrow \infty$, we simplify the functions

$$
\begin{gathered}
C_{1}\left(a, b, \tau_{M, a, b}\right)=(1+o(1)) \frac{t_{b}^{\prime 2}}{M a}, \\
C_{2}\left(a, \tilde{b}, r_{M, a, \tilde{b}}\right)=\frac{\sigma_{T}(M a-\tilde{b})^{3}}{(a-\tilde{b})^{2} \sigma_{T}^{3}}(1+o(1))=\frac{M^{3} a}{\sigma_{T}^{2}}(1+o(1)) .
\end{gathered}
$$

We now show that the second term in (22) is of a smaller order, that is,

$$
\left(1-\Phi\left(r_{M, a, \tilde{b}}+t_{W_{\tilde{b}}}\right)\right) C_{2}\left(a, \tilde{b}, r_{M, a, \tilde{b}}\right)=o(1)\left(1-\Phi\left(\tau_{M, a, b}+t_{b}\right)\right) C_{1}\left(a, b, \tau_{M, a, b}\right) .
$$

By choosing $\tilde{b}$ as a constant and sending $a$ to $\infty$, for some $\lambda>0$, we have

$$
\begin{aligned}
\left(r_{M, a, \tilde{b}}+t_{W_{\tilde{b}}}\right)^{2}-\left(t_{b}+\tau_{M, a, b}\right)^{2} & \\
& =\left(\frac{M a-\tilde{b}}{\sigma_{T}}+t_{W_{\tilde{b}}}\right)^{2}-\left(t_{b}+\frac{a-b}{M a}\left(\frac{a-b}{M a}+1\right) t_{b}^{\prime}\right)^{2} \\
& \geq(2+o(1)) a\left(\frac{M a-\tilde{b}}{\sigma_{T}}+t_{W_{\tilde{b}}}-t_{b}-\frac{a-b}{M a}\left(\frac{a-b}{M a}+1\right) t_{b}^{\prime}\right) \\
& \geq(2+o(1)) a\left(\frac{M a}{\sigma_{T}}-t_{b}+\left(t_{W_{\tilde{b}}}-\sigma_{T}^{-1} \tilde{b}\right)+o(1)\right) \\
& \geq(2+o(1)) \frac{\lambda}{2 \sigma_{T}} a,
\end{aligned}
$$

where the second inequality follows from the following argument. By the fact that $t_{W_{b}} \leq t_{b}^{\prime} \leq t_{b}$, we have

$$
\begin{aligned}
\frac{a-b}{M a}\left(\frac{a-b}{M a}+1\right) t_{b}^{\prime} & =\left(1+\frac{a-b}{\sigma_{T}(a+C) t_{b} / b}\right) \frac{a-b}{\sigma_{T}(a+C) t_{b} / b} t_{b}^{\prime} \\
& =(1+o(1)) \frac{a-b}{\sigma_{T} t_{b}} t_{b}^{\prime} \\
& =o(1) .
\end{aligned}
$$

Therefore, we obtain (23), and the second term in (22) can be ignored. Furthermore, by (24), 
we have

$$
\begin{aligned}
\left(t_{b}+\tau_{M, a, b}\right)^{2} & =\left(t_{b}+\left(1+\frac{a-b}{\sigma_{T}(a+C) t_{b} / b}\right) \frac{a-b}{\sigma_{T}(a+C) t_{b} / b} t_{b}^{\prime}\right)^{2} \\
& \geq t_{b}^{2}+2 \frac{(a-b) b}{\sigma_{T}(a+C)} t_{b}^{\prime} \\
& \geq t_{b}^{2}+2 \frac{(a-b) b}{\sigma_{T}^{2}}+o(1),
\end{aligned}
$$

where the last step follows from the fact that $t_{b}^{\prime} \geq t_{W_{b}}=\sigma_{T}^{-1} b+O(1)$. Therefore,

$$
\begin{aligned}
(1- & \left.\Phi\left(\tau_{M, a, b}+t_{b}\right)\right) C_{1}\left(a, b, \tau_{M, a, b}\right) \\
& \leq(1+o(1)) \frac{t_{b}^{\prime 2}}{M a} \frac{1}{\sqrt{2 \pi}} \frac{1}{\tau_{M, a, b}+t_{b}} \exp \left(-\frac{t_{b}^{2}+2(a-b) b / \sigma_{T}^{2}}{2}\right) \\
& \leq(1+o(1)) \frac{1}{\sqrt{2 \pi} \sigma_{T}} \exp \left(-\frac{t_{b}^{2}+2(a-b) b / \sigma_{T}^{2}}{2}\right),
\end{aligned}
$$

where the last inequality follows from the fact that $t_{b}^{\prime}<t_{b}<t_{b}+\tau_{M, a, b}$ and $t_{b}^{\prime}<M a / \sigma_{T}=$ $(a+C) t_{b} / b$. This completes the proof of Proposition 1 .

\subsection{Proofs of the theorems}

In this section we prove our theorems based on Proposition 1. We propose a change of measure $\mathbb{Q}$ which is central to the proof of our theorems. Let $\mathbb{P}$ be the original measure. The probability measure $\mathbb{Q}$ is defined such that $\mathbb{P}$ and $\mathbb{Q}$ are mutually absolutely continuous with the Radon-Nikodym derivative being

$$
\frac{\mathrm{d} \mathbb{Q}}{\mathrm{dP}}=\int_{T} \frac{\exp \left(-(f(t)-u)^{2} / 2\right)}{\exp \left(-f(t)^{2} / 2\right)} \mathrm{d} \vartheta(t)
$$

for some $u \in \mathbb{R}$. Note that $\mathbb{Q}$ depends on $u$. To simplify the notation, we omit the index of $u$ in $\mathbb{Q}$ when there is no ambiguity. One can verify that (25) is a valid Radon-Nikodym derivative. We will provide further description in Section 3.2.2. See also [14], where this change of measure was used to derive the asymptotic approximation of $\mathbb{P}\left(\int_{T} \exp \left(\sigma f(t)+\mu_{f}(t)\right) \mathrm{d} t>b\right)$ with $\mu_{f}(t)$ being a deterministic function.

3.2.1. Proof of Theorem 1. In order to use Proposition 1, we first derive a lower bound for $t_{a}$. For each $u$, we rewrite

$$
\frac{\mathrm{d} \mathbb{Q}}{\mathrm{d} \mathbb{P}}=\int_{T} \exp \left(\frac{2 u f(t)-u^{2}}{2}\right) \mathrm{d} \vartheta(t) .
$$

We have

$$
\begin{aligned}
\mathbb{P}\left(\log \int_{T} \mathrm{e}^{\sigma(t) f(t)} \mathrm{d} \vartheta(t)>a\right) & =\mathbb{E}_{\mathbb{Q}}\left(\frac{\mathrm{d} \mathbb{P}}{\mathrm{d} \mathbb{Q}} ; \log \int_{T} \mathrm{e}^{\sigma(t) f(t)} \mathrm{d} \vartheta(t)>a\right) \\
& =\mathrm{e}^{u^{2} / 2} \mathbb{E}_{\mathbb{Q}}\left(\frac{1}{\int_{T} \mathrm{e}^{u f(t)} \mathrm{d} \vartheta(t)} ; \log \int_{T} \mathrm{e}^{\sigma(t) f(t)} \mathrm{d} \vartheta(t)>a\right),
\end{aligned}
$$

where $\mathbb{E}_{\mathbb{Q}}$ is the expectation under measure $\mathbb{Q}$. Note that

$$
\log \int_{T} \mathrm{e}^{\sigma(t) f(t)} \mathrm{d} \vartheta(t)>a
$$


implies that, for large enough $a$,

$$
\begin{aligned}
\int_{T} \mathrm{e}^{\sigma_{T} f(t)} \mathrm{d} \vartheta(t) & \geq \int_{T \cap\{f(t) \geq 0\}} \mathrm{e}^{\sigma(t) f(t)} \mathrm{d} \vartheta(t) \\
& \geq \mathrm{e}^{a}-\int_{T \cap\{f(t)<0\}} \mathrm{e}^{\sigma(t) f(t)} \mathrm{d} \vartheta(t) \\
& \geq \mathrm{e}^{a}-1
\end{aligned}
$$

Then by Jensen's inequality we have, conditioning on $\log \int_{T} \mathrm{e}^{\sigma(t) f(t)} \mathrm{d} \vartheta(t)>a$ with large enough $a$,

$$
\int_{T} \mathrm{e}^{u f(t)} \mathrm{d} \vartheta(t) \geq\left(\mathrm{e}^{a}-1\right)^{u / \sigma_{T}}
$$

and, therefore,

$$
\begin{aligned}
\mathbb{P}\left(\log \int_{T} \mathrm{e}^{\sigma(t) f(t)} \mathrm{d} \vartheta(t)>a\right) & =\mathrm{e}^{u^{2} / 2} \mathbb{E}_{\mathbb{Q}}\left(\frac{1}{\int_{T} \mathrm{e}^{u f(t)} \mathrm{d} \vartheta(t)} ; \log \int_{T} \mathrm{e}^{\sigma(t) f(t)} \mathrm{d} \vartheta(t)>a\right) \\
& \leq \mathrm{e}^{u^{2} / 2}\left(\mathrm{e}^{a}-1\right)^{-u / \sigma_{T}} \\
& =\left(1-\mathrm{e}^{-a}\right)^{-u / \sigma_{T}} \mathrm{e}^{u^{2} / 2-u a / \sigma_{T}} .
\end{aligned}
$$

This bound holds for all $u$, and $\exp \left(u^{2} / 2-u a / \sigma_{T}\right)$ is minimized when $u=a / \sigma$. Thus, for sufficiently large $a$, the bound of the tail is

$$
1-F(a)=\mathbb{P}\left(\log \int_{T} \mathrm{e}^{\sigma(t) f(t)} \mathrm{d} \vartheta(t)>a\right) \leq(1+o(1)) \exp \left(-\frac{a^{2}}{2 \sigma_{T}^{2}}\right) .
$$

According to the above inequality, we have

$$
t_{a} \geq \Phi^{-1}\left(1-\exp \left(-\frac{a^{2}}{2 \sigma_{T}^{2}}\right)\right)=\frac{a}{\sigma_{T}}-\sigma_{T} \frac{\log a-\log \sigma_{T}}{a}+\frac{\tilde{C}}{a}+o\left(\frac{1}{a}\right),
$$

where $\tilde{C}$ satisfies $(1 / \sqrt{2 \pi}) \exp \left(-\tilde{C} / \sigma_{T}\right)=1$. Then, by Proposition 1 , taking $b=a-1 / \sqrt{a}$, we have

$$
\begin{aligned}
F^{\prime}(a) & \leq(1+o(1)) \frac{1}{\sqrt{2 \pi} \sigma_{T}} \exp \left(-\frac{t_{b}^{2}+2(a-b) b / \sigma_{T}^{2}}{2}\right) \\
& \leq(1+o(1)) \frac{a}{\sigma_{T}^{2}} \exp \left(-\frac{a^{2}}{2 \sigma_{T}^{2}}\right),
\end{aligned}
$$

which completes our proof.

3.2.2. Proof of Theorem 2. Under the assumptions of Theorem 2, $\sigma(t) \equiv \sigma$ and $\mathrm{d} \vartheta(t)=$ $\mathrm{e}^{\mu_{f}(t)} \mathrm{d} t / \int_{T} \mathrm{e}^{\mu_{f}(t)} \mathrm{d} t$. Then

$$
\mathbb{P}\left(\log \int_{T} \mathrm{e}^{\sigma f(t)} \mathrm{d} \vartheta(t)>a\right)=\mathbb{P}\left(\log \int_{T} \mathrm{e}^{\sigma f(t)+\mu_{f}(t)} \mathrm{d} t>a+\log \int_{T} \mathrm{e}^{\mu_{f}(t)} \mathrm{d} t\right) .
$$

Similarly to the proof of Theorem 1, we prove Theorem 2 by deriving an upper bound for

$$
\mathbb{P}\left(\log \int_{T} \mathrm{e}^{\sigma f(t)+\mu_{f}(t)} \mathrm{d} t>a\right),
$$

which helps to get a lower bound for $t_{a}$ (then replace $a$ by $a+\log \int \mathrm{e}^{\mu_{f}(t)} \mathrm{d} t$ ). 
Consider the change of measure:

$$
\frac{\mathrm{d} \mathbb{Q}}{\mathrm{d} \mathbb{P}}=\frac{1}{\operatorname{mes}(T)} \int_{T} \frac{\exp \left(-(f(t)-u)^{2} / 2\right)}{\exp \left(-f(t)^{2} / 2\right)} \mathrm{d} t=\frac{1}{\operatorname{mes}(T)} \int_{T} \exp \left(\frac{2 u f(t)-u^{2}}{2}\right) \mathrm{d} t,
$$

where mes $(T)$ is the Lebesgue measure of $T$. It is more intuitive to describe the measure $\mathbb{Q}$ from a simulation point of view (see [14]). One can simulate $f(t)$ under the measure $\mathbb{Q}$ according to the following two steps.

1. Simulate a random variable $\tau$ uniformly over $T$ with respect to the Lebesgue measure.

2. Given the realized $\tau$, simulate the Gaussian process $f(t)$ with mean $u C(t-\tau)$ and covariance function $C(t)$.

The second step is equivalent to first sampling $f(\tau)$ from $N(u, 1)$ and then sampling $\{f(t): t \neq$ $\tau$ \} from its original conditional distribution under the measure $\mathbb{P}$ given $f(\tau)$. It is not hard to verify that the above two-step procedure is consistent with the Radon-Nikodym derivative in (27). Under $\mathbb{Q}$, a random variable $\tau$ is first sampled uniformly over $T$, then $f(\tau)$ is simulated with a large mean at level $u$. This implies that the high value of the integral $\int_{T} \mathrm{e}^{\sigma f(t)} \mathrm{d} t$ is mostly caused by the fact that the field reaches a high level at one location $t^{*}$, and such a location $t^{*}$ is very close to $\tau$. Therefore, the random index $\tau$ localizes the maximum of the field. We can write the tail probability as

$$
\begin{aligned}
& \mathbb{P}\left(\log \int_{T} \mathrm{e}^{\sigma f(t)+\mu_{f}(t)} \mathrm{d} t>a\right) \\
& =\operatorname{mes}(T) \mathrm{e}^{u^{2} / 2} \mathbb{E}_{\mathbb{Q}}\left(\frac{1}{\int_{T} \mathrm{e}^{u f(t)} \mathrm{d} t} ; \log \int_{T} \mathrm{e}^{\sigma f(t)+\mu_{f}(t)} \mathrm{d} t>a\right) \\
& =\mathrm{e}^{u^{2} / 2} \int_{T} \mathbb{E}_{\mathbb{Q}}\left(\frac{1}{\int_{T} \mathrm{e}^{u f(t)} \mathrm{d} t} ; \log \int_{T} \mathrm{e}^{\sigma f(t)+\mu_{f}(t)} \mathrm{d} t>a \mid \tau\right) \mathrm{d} \tau .
\end{aligned}
$$

According to step 2 of the simulation, conditional on $\tau$ and under the measure $\mathbb{Q}$, the process

$$
\bar{f}(t)=f(t)-u C(t-\tau)
$$

follows the same law as $f(t)$ under $\mathbb{P}$.

Let $u$ be the solution to $\mathrm{e}^{a-\sigma u} u^{d / 2 \alpha-d \gamma}=1$ with $0<\gamma<\varepsilon$, where $\varepsilon$ is chosen as in the theorem statement. Choose $\delta$ such that $\mathrm{e}^{-\sup _{t \in T} \mu_{f}(t)} u^{d / 2 \alpha+d \gamma}=\operatorname{mes}\left(s \in T:|s|<u^{\delta}\right)$. Keep in mind that $\delta \approx 1 / 2 \alpha+\gamma$. Let

$$
\mathcal{L}=\left\{\sup _{t \in T} \bar{f}(t)<a^{1 / 2+\eta}\right\} .
$$

For any $\eta$ satisfying $0<\eta<\alpha \delta-\frac{1}{2}$, by Jensen's inequality we have (28) on $\mathcal{L}^{c}$ :

$$
\begin{aligned}
& \mathbb{P}\left(\log \int_{T} \mathrm{e}^{\sigma f(t)+\mu_{f}(t)} \mathrm{d} t>a, \mathcal{L}^{c}\right) \\
& \quad=\operatorname{mes}(T) \mathrm{e}^{u^{2} / 2} \mathbb{E}_{\mathbb{Q}}\left(\frac{1}{\int_{T} \mathrm{e}^{u f(t)} \mathrm{d} t} ; \log \int_{T} \mathrm{e}^{\sigma f(t)} \mathrm{d} t>a-\sup _{t \in T} \mu_{f}(t) \text { and } \mathcal{L}^{c}\right)
\end{aligned}
$$




$$
\begin{aligned}
\leq & \exp \left(\frac{u^{2}}{2}-\frac{u\left(a-\sup _{t \in T} \mu_{f}(t)-\log \operatorname{mes}(T)\right)}{\sigma}\right) \\
& \times \mathbb{Q}\left(\log \int_{T} \mathrm{e}^{\sigma f(t)} \mathrm{d} t>a-\sup _{t \in T} \mu_{f}(t) \text { and } \sup _{t \in T} \bar{f}(t)>a^{1 / 2+\eta}\right) \\
= & o(1) \mathrm{e}^{-u^{2} / 2} .
\end{aligned}
$$

Here the inequality follows thanks to (26) and the last step follows from the Borel-TIS lemma (applied to $\bar{f}$ ). Therefore, we have

$$
\begin{aligned}
& \mathbb{P}\left(\log \int_{T} \mathrm{e}^{\sigma f(t)+\mu_{f}(t)} \mathrm{d} t>a\right) \\
& \leq \leq \mathrm{e}^{u^{2} / 2} \int_{T} \mathbb{E}_{\mathbb{Q}}\left(\frac{1}{\int_{T} \mathrm{e}^{u f(t)} \mathrm{d} t} ; \log \int_{T} \mathrm{e}^{\sigma f(t)} \mathrm{d} t>a-\sup _{t \in T} \mu_{f}(t) \text { and } \mathcal{L} \mid \tau\right) \mathrm{d} \tau \\
& \quad+o(1) \mathrm{e}^{-u^{2} / 2}
\end{aligned}
$$

In what follows, we derive an upper bound for the conditional expectation in (29). We first consider the set $\left\{\log \int_{T} \mathrm{e}^{\sigma f(t)} \mathrm{d} t>a-\sup _{t \in T} \mu_{f}(t)\right\}$ in (29). Let $\varepsilon_{u}=u^{-1 / \alpha+\delta}$ (recall that $\delta$ is some constant such that $\left.\mathrm{e}^{-\sup _{t \in T} \mu_{f}(t)} u^{d / 2 \alpha+d \gamma}=\operatorname{mes}\left(s \in T:|s|<u^{\delta}\right)\right)$. We can split the integral $\log \int_{T} \mathrm{e}^{\sigma f(t)} \mathrm{d} t$ into two parts:

$$
\int_{T} \mathrm{e}^{\sigma f(t)} \mathrm{d} t=\mathrm{e}^{\sigma u} T_{1}+\mathrm{e}^{\sigma u} T_{2},
$$

where

$$
T_{1}=\int_{|t-\tau|<\varepsilon_{u}} \mathrm{e}^{\sigma f(t)} \mathrm{d} t=\int_{|t-\tau|<\varepsilon_{u}} \mathrm{e}^{\sigma \bar{f}(t)+\sigma u(C(t-\tau)-C(0))} \mathrm{d} t
$$

and

$$
T_{2}=\int_{|t-\tau| \geq \varepsilon_{u}} \mathrm{e}^{\sigma f(t)} \mathrm{d} t=\int_{|t-\tau| \geq \varepsilon_{u}} \mathrm{e}^{\sigma \bar{f}(t)+\sigma u(C(t-\tau)-C(0))} \mathrm{d} t .
$$

Thus, $\log \int \mathrm{e}^{\sigma f(t)} \mathrm{d} t>a-\sup _{t \in T} \mu_{f}(t)$ if and only if

$$
T_{1}+T_{2}>\mathrm{e}^{-\sup _{t \in T} \mu_{f}(t)} u^{d \gamma-d / 2 \alpha} .
$$

For $T_{1}$, since $C(0)-C(t-\tau)=|t-\tau|^{\alpha}+R(t-\tau)$, where $R(t-\tau)=o\left(|t-\tau|^{\alpha}\right)$,

$$
\begin{aligned}
T_{1} & =\int_{|t-\tau|<\varepsilon_{u}} \mathrm{e}^{\sigma \bar{f}(t)+\sigma u(C(t-\tau)-C(0))} \mathrm{d} t \\
& =\int_{|t-\tau|<\varepsilon_{u}} \exp \left(\sigma \bar{f}(t)-\sigma u\left(|t-\tau|^{\alpha}+R(t-\tau)\right)\right) \mathrm{d} t \\
& =u^{-d / \alpha} \int_{|s|<u^{\delta}} \exp \left(\sigma \bar{f}\left(\tau+u^{-1 / \alpha} s\right)-\sigma|s|^{\alpha}-u \sigma R\left(u^{-1 / \alpha} s\right)\right) \mathrm{d} s .
\end{aligned}
$$

For $T_{2}$, by the condition that $\sup _{t \in T \backslash \mathcal{N}_{0}} C(t)<C(0)=1$, where $\mathcal{N}_{0}$ is a neighborhood of 0 , we have, for large enough $u$,

$$
T_{2} \leq \mathrm{e}^{-\sigma u^{\delta \alpha}} \int_{T} \mathrm{e}^{\sigma \bar{f}(t)} \mathrm{d} t
$$


and on the set $\mathcal{L}$ we have

$$
T_{2}<\operatorname{mes}(T) \mathrm{e}^{\sigma a^{1 / 2+\eta}-\sigma u^{\delta \alpha}} .
$$

For the term $\int_{T} \mathrm{e}^{u f(t)} \mathrm{d} t$ in (29), we have

$$
\begin{aligned}
\int_{|t-\tau|<\varepsilon_{u}} \mathrm{e}^{u f(t)} \mathrm{d} t= & \mathrm{e}^{u^{2}} \int_{|t-\tau|<\varepsilon_{u}} \mathrm{e}^{u(\bar{f}(t)+u(C(t-\tau)-C(0)))} \mathrm{d} t \\
= & \mathrm{e}^{u^{2}} u^{-d / \alpha} \operatorname{mes}\left(|s|<u^{\delta}\right) \frac{1}{\operatorname{mes}\left(|s|<u^{\delta}\right)} \\
& \times \int_{|s|<u^{\delta}} \exp \left(u\left(\bar{f}\left(\tau+u^{-1 / \alpha} s\right)-|s|^{\alpha}-u R\left(u^{-1 / \alpha} s\right)\right)\right) \mathrm{d} s .
\end{aligned}
$$

By Jensen's inequality and (30), on the set $\left\{\log \int \mathrm{e}^{\sigma f(t)} \mathrm{d} t>a\right\}$, we have

$$
\begin{aligned}
\frac{1}{\operatorname{mes}\left(|s|<u^{\delta}\right)} \int_{|s|<u^{\delta}} \exp \left(u\left(\bar{f}\left(\tau+u^{-1 / \alpha} s\right)-|s|^{\alpha}-u R\left(u^{-1 / \alpha} s\right)\right)\right) \mathrm{d} s \\
\quad \geq\left(\frac{1}{\operatorname{mes}\left(|s|<u^{\delta}\right)} \int_{|s|<u^{\delta}} \exp \left(\sigma \bar{f}\left(\tau+u^{-1 / \alpha} s\right)-\sigma|s|^{\alpha}-\sigma u R\left(u^{-1 / \alpha} s\right)\right) \mathrm{d} s\right)^{u / \sigma} \\
\quad=\left(\frac{u^{d / \alpha} T_{1}}{\operatorname{mes}\left(|s| \leq u^{\delta}\right)}\right)^{u / \sigma} \\
\quad=\left(\frac{u^{d / \alpha} T_{1}}{\left.\mathrm{e}^{-\sup _{t \in T} \mu_{f}^{(t)}} u^{d / 2 \alpha+d \gamma}\right)^{u / \sigma}}\right. \\
\geq\left(1-\mathrm{e}^{\sup _{t \in T} \mu_{f}(t)} u^{d / 2 \alpha-d \gamma} T_{2}\right)^{u / \sigma} \\
\geq\left(1-\mathrm{e}^{\sup _{t \in T} \mu_{f}(t)} u^{d / 2 \alpha-d \gamma} \operatorname{mes}(T) \mathrm{e}^{\sigma a^{1 / 2+\eta}-\sigma u^{\delta \alpha}}\right)^{u / \sigma}
\end{aligned}
$$

The first equality in the above display is due to (31); the second equality is due to the definition of $\delta$; the second inequality is due to (30); and the last step is due to (32). Now, combining (31), (32), (33), and (34), we obtain

$$
\begin{aligned}
& \mathbb{E}_{\mathbb{Q}}\left(\frac{1}{\int_{T} \mathrm{e}^{u f(t)} \mathrm{d} t} ; \log \int_{T} \mathrm{e}^{\sigma f(t)} \mathrm{d} t>a-\sup _{t \in T} \mu_{f}(t) \text { and } \mathcal{L} \mid \tau\right) \\
& \leq \mathbb{E}_{\mathbb{Q}}\left(\frac{1}{\mathrm{e}^{u^{2}} \mathrm{e}^{-\sup _{t \in T} \mu_{f}(t)} u^{-d / 2 \alpha+d \gamma}\left(1-\operatorname{mes}(T) \mathrm{e}^{\sup _{t \in T} \mu_{f}(t)} u^{d / 2 \alpha-d \gamma} \mathrm{e}^{\sigma a^{1 / 2+\eta}-\sigma u^{\delta \alpha}}\right)^{u / \sigma}} ;\right. \\
& \left.u^{d / 2 \alpha-d \gamma} T_{1}>1-\operatorname{mes}(T) \mathrm{e}^{\sup _{t \in T} \mu_{f}(t)} u^{d / 2 \alpha-d \gamma} \mathrm{e}^{\sigma a^{1 / 2+\eta}-\sigma u^{\delta \alpha}} \text { and } \mathcal{L}\right) \\
& \leq(1+o(1)) \mathrm{e}^{\sup _{t \in T} \mu_{f}(t)} u^{d / 2 \alpha-d \gamma} \mathrm{e}^{-u^{2}} .
\end{aligned}
$$

Note that $u$ is the solution to $\mathrm{e}^{a-\sigma u} u^{d / 2 \alpha-d \gamma}=1$. Then following (29) we can obtain

$$
\mathbb{P}\left(\log \int_{T} \mathrm{e}^{\sigma f(t)} \mathrm{d} t>a\right) \leq(\operatorname{mes}(T)+o(1)) \mathrm{e}^{\sup _{t \in T} \mu_{f}(t)} u^{d / 2 \alpha-d \gamma} \mathrm{e}^{-u^{2} / 2},
$$

which implies that

$$
\begin{aligned}
1-F(a) & =\mathbb{P}\left(\log \int_{T} \mathrm{e}^{\sigma f(t)+\mu_{f}(t)} \mathrm{d} t>a+\log \int_{T} \mathrm{e}^{\mu_{f}(t)} \mathrm{d} t\right) \\
& \leq(\operatorname{mes}(T)+o(1)) \mathrm{e}^{\sup _{t \in T} \mu_{f}(t)} u_{\gamma}^{d / 2 \alpha-d \gamma} \mathrm{e}^{-u_{\gamma}{ }^{2} / 2},
\end{aligned}
$$


where $u_{\gamma}$ is the solution to

$$
\int_{T} \mathrm{e}^{\mu_{f}(t)} \mathrm{d} t \mathrm{e}^{a-\sigma u_{\gamma}} u_{\gamma}^{d / 2 \alpha-d \gamma}=1
$$

Then

$$
\begin{aligned}
t_{a} & \geq \Phi^{-1}\left(1-\operatorname{mes}(T) \mathrm{e}^{\sup _{t \in T} \mu_{f}(t)} u_{\gamma}^{d / 2 \alpha-d \gamma} \mathrm{e}^{-u_{\gamma}^{2} / 2}\right) \\
& =u_{\gamma}-\frac{(d / 2 \alpha-d \gamma+1) \log u_{\gamma}}{u_{\gamma}}-\frac{\log \left(\sqrt{2 \pi} \operatorname{mes}(T) \mathrm{e}^{\sup _{t \in T} \mu_{f}(t)}\right)}{u_{\gamma}}+o\left(\frac{1}{a}\right) .
\end{aligned}
$$

Therefore, by Proposition 1 , taking $b=a-1 / \sqrt{a}$, we have

$$
F^{\prime}(a) \leq(\operatorname{mes}(T)+o(1)) \mathrm{e}^{\sup _{t \in T} \mu_{f}(t)} \sigma^{-1} u_{\gamma}^{1+d / 2 \alpha-d \gamma} \mathrm{e}^{-u_{\gamma}^{2} / 2}
$$

Then, for any $\varepsilon \in(0,1 / 2 \alpha)$, taking $\gamma$ such that $\gamma<\varepsilon$, we have

$$
\limsup _{a \rightarrow \infty} u_{\varepsilon}^{d \varepsilon-d / 2 \alpha-1} \mathrm{e}^{u_{\varepsilon}^{2} / 2} F^{\prime}(a)=0,
$$

which completes the proof of Theorem 2.

3.2.3. Proof of Theorem 3. We cite the following result (see Theorem 3.4 of [14]) that provides an approximation of $F(a)$ for three-time differentiable fields.

Lemma 7. Under the assumptions and notation of Theorem 3,

$$
\begin{aligned}
& \mathbb{P}\left(\log \int_{T} \mathrm{e}^{\sigma f(t)+\mu_{f}(t)} \mathrm{d} t>a\right) \\
& \quad=(1+o(1)) u^{d-1} \int_{T} \exp \left(-\frac{\left(u-\mu_{f}(t) / \sigma\right)^{2}}{2}\right) C_{H}\left(\mu_{f}, \sigma, t\right) \mathrm{d} t,
\end{aligned}
$$

where $u$ is the solution to

$$
\left(\frac{2 \pi}{\sigma}\right)^{d / 2} u^{-d / 2} \mathrm{e}^{\sigma u}=\mathrm{e}^{a}
$$

By Lemma 7, for a three times differentiable Gaussian random field satisfying the conditions in Theorem 3, we have

$$
\begin{aligned}
1-F(a) & =\mathbb{P}\left(\log \int_{T} \mathrm{e}^{\sigma f(t)+\mu_{f}(t)} \mathrm{d} t>a+\log \int_{T} \mathrm{e}^{\mu_{f}(t)} \mathrm{d} t\right) \\
& =(1+o(1)) \tilde{u}^{d-1} \int_{T} \exp \left(-\frac{\left(\tilde{u}-\mu_{f}(t) / \sigma\right)^{2}}{2}\right) C_{H}\left(\mu_{f}, \sigma, t\right) \mathrm{d} t
\end{aligned}
$$

where $\tilde{u}$ is the solution to

$$
\left(\frac{2 \pi}{\sigma}\right)^{d / 2} \tilde{u}^{-d / 2} \mathrm{e}^{\sigma \tilde{u}}=\mathrm{e}^{a} \int_{T} \mathrm{e}^{\mu_{f}(t)} \mathrm{d} t
$$

Therefore, we obtain

$$
t_{a}=\Phi^{-1}\left(\tilde{u}^{d-1} \int_{T} \exp \left(-\frac{\left(\tilde{u}-\mu_{f}(t) / \sigma\right)^{2}}{2}\right) C_{H}\left(\mu_{f}, \sigma, t\right) \mathrm{d} t\right)+o\left(\frac{1}{a}\right)
$$


which implies that $t_{a} / a \rightarrow \sigma^{-1}$. Then, by Proposition 1 , letting $b=a-1 / \sqrt{a}$, we have

$$
F^{\prime}(a) \leq(1+o(1)) \sigma^{-2} a(1-F(a)) .
$$

The right-hand side of the above display is precisely the approximation in the theorem.

In order to prove the theorem, we need to show that the right-hand side of the above equality is also an asymptotic lower bound of the density. According to the approximation in (35), we have

$$
1-F(a)=\int_{a}^{\infty} F^{\prime}(x) \mathrm{d} x \leq(1+o(1)) \int_{a}^{\infty} \sigma^{-2} x(1-F(x)) \mathrm{d} x=(1+o(1))(1-F(a)) .
$$

We prove the lower bound by reaching a contradiction to (36). If our conclusion does not hold, there exists $\varepsilon>0$ and $\left\{a_{i}, i \geq 1\right\}$ such that $\lim _{i} a_{i} \rightarrow \infty$ and

$$
\frac{F^{\prime}\left(a_{i}\right)}{\sigma^{-2} a_{i}\left(1-F\left(a_{i}\right)\right)}<1-\varepsilon
$$

Then

$$
\int_{a_{i}}^{\infty}\left[\sigma^{-2} x(1-F(x))-F^{\prime}(x)\right] \mathrm{d} x \geq(1+o(1)) \int_{a_{i}}^{\tilde{a}_{i}} \frac{\varepsilon}{2} \sigma^{-2} x(1-F(x)) \mathrm{d} x,
$$

where

$$
\tilde{a}_{i}=\inf \left\{x>a_{i}: \sigma^{-2} x(1-F(x))-F^{\prime}(x)>\frac{1}{2} \varepsilon \sigma^{-2} a_{i}\left(1-F\left(a_{i}\right)\right)\right\} .
$$

We have a lower bound for $\tilde{a}_{i}$ as

$$
\tilde{a}_{i} \geq a_{i}+\frac{(\varepsilon / 2) \sigma^{-2} a_{i}\left(1-F\left(a_{i}\right)\right)}{\sup _{a \geq a_{i}} D^{+} F^{\prime}(a)+\left|\partial \sigma^{-2} a(1-F(a)) / \partial a\right|_{a=a_{i}} \mid} .
$$

Following the result in Lemma 8 (see Appendix A), we derive an upper bound for $D^{+} F^{\prime}\left(a_{i}\right)$ as in steps 3 and 4 in the last section. Under the conditions of this theorem, we have $M=$ $\sigma(1+C / a) t_{a} / a \rightarrow 1$; then, for $b=a-1 / \sqrt{a}$,

$$
\begin{aligned}
D^{+} F^{\prime}(a) \leq & \left(1-\Phi\left(\tau_{M, a, b}+t_{b}\right)\right) C_{3}\left(a, b, r_{M, a, b}\right) \\
& +\left(1-\Phi\left(r_{M, a, b}+t_{W_{b}}\right)\right) C_{4}\left(a, b, r_{M, a, b}\right) \\
= & (1+o(1))\left(1-\Phi\left(\tau_{M, a, b}+t_{b}\right)\right) C_{3}\left(a, b, r_{M, a, b}\right) \\
= & (1+o(1)) \sigma^{-4} a^{2}\left(1-\Phi\left(\tau_{M, a, b}+t_{b}\right)\right) \\
= & (1+o(1)) \sigma^{-4} a^{2}(1-F(a)) .
\end{aligned}
$$

Therefore,

$$
\tilde{a}_{i} \geq a_{i}+(1+o(1)) \frac{(\varepsilon / 2) \sigma^{-2} a_{i}\left(1-F\left(a_{i}\right)\right)}{2 \sigma^{-4} a_{i}^{2}\left(1-F\left(a_{i}\right)\right)}=a_{i}+(1+o(1)) \frac{\varepsilon \sigma^{2}}{4 a_{i}} .
$$

Thus, for the right-hand side integral in (37), we have

$$
(1+o(1)) \int_{a_{i}}^{\tilde{a}_{i}} \frac{\varepsilon}{2} \sigma^{-2} x(1-F(x)) \mathrm{d} x \geq(1+o(1)) \eta_{\varepsilon}\left(1-F\left(a_{i}\right)\right)
$$


where $\eta_{\varepsilon}>0$ depends on $\varepsilon$ and $\sigma$. Then (37) and (38) indicate that, for all $a_{i}$,

$$
\int_{a_{i}}^{\infty}\left[\sigma^{-2} x(1-F(x))-F^{\prime}(x)\right] \mathrm{d} x \geq(1+o(1)) \eta_{\varepsilon}\left(1-F\left(a_{i}\right)\right) .
$$

This contradicts the fact (implied by (36)) that

$$
\int_{a}^{\infty}\left[\sigma^{-2} x(1-F(x))-F^{\prime}(x)\right] \mathrm{d} x=o(1-F(a)) .
$$

This completes the proof.

\section{Appendix A. Proofs of the lemmas}

Proof of Lemma 1. Lemma 1 follows from a similar argument as in [20]. For (9), the inequality

$$
\exp \left(-\frac{t^{2}}{2}\right) \leq\left(t_{+}+1\right) \int_{t}^{\infty} \exp \left(-\frac{u^{2}}{2}\right) \mathrm{d} u
$$

implies that

$$
\begin{aligned}
\int_{S_{a}} l_{x} & \varphi_{N}(x) \mathrm{d} S_{a}(x) \\
& =\int_{S_{a}} l_{x}(2 \pi)^{-N / 2} \exp \left(-\frac{|x|^{2}-c_{x}^{2}}{2}\right) \exp \left(-\frac{c_{x}^{2}}{2}\right) \mathrm{d} S_{a}(x) \\
& \leq \int_{S_{a}} l_{x}(2 \pi)^{-N / 2} \exp \left(-\frac{|x|^{2}-c_{x}^{2}}{2}\right)\left(c_{x}^{+}+1\right) \int_{c_{x}}^{\infty} \exp \left(-\frac{u^{2}}{2}\right) \mathrm{d} u \mathrm{~d} S_{a}(x) \\
& =\int_{S_{a}} l_{x}\left(c_{x}^{+}+1\right) \int_{0}^{\infty} \varphi_{N}\left(x+\lambda \boldsymbol{n}_{x}\right) \mathrm{d} \lambda \mathrm{d} S_{a}(x) .
\end{aligned}
$$

The last step is due to a change of variable $u=c_{x}+\lambda$ and the fact that

$$
\left|x+\lambda n_{x}\right|^{2}=|x|^{2}+\lambda^{2}+2 \lambda c_{x} .
$$

The above surface integral can be bounded by a volume integral, i.e.

$$
\begin{aligned}
\int_{S_{a}} l_{x}\left(c_{x}^{+}+1\right) \int_{0}^{\infty} \varphi_{N}\left(x+\lambda \boldsymbol{n}_{x}\right) \mathrm{d} \lambda \mathrm{d} S_{a}(x) \\
\quad \leq \int_{S_{a}} \int_{0}^{\infty} l_{x}\left(c_{x}^{+}+1\right) \prod_{i=1}^{N-1}\left(1+\lambda k_{i}(x)\right) \varphi_{N}\left(x+\lambda \boldsymbol{n}_{x}\right) \mathrm{d} \lambda \mathrm{d} S_{a}(x) \\
\quad=\int_{V_{N, a}^{c}} l_{h(x)}\left(c_{h(x)}^{+}+1\right) \varphi_{N}(x) \mathrm{d} x
\end{aligned}
$$

where the $k_{i}(x)$ are the principle curvatures of $S_{a}$ at $x$. The above inequality results from the fact that curvatures are nonnegative in that $S_{a}$ is the border of a convex set. Therefore, we obtain

$$
F_{N}^{\prime}(a) \leq \int_{V_{N, a}^{c}} l_{h(x)}\left(c_{h(x)}^{+}+1\right) \mathrm{d} \mu_{N}(x) .
$$


Proof of Lemma 2. For any two functions $f$ and $g$, if $\log \left(\int_{T} \exp (f(t)) \mathrm{d} \vartheta(t)\right) \leq a$ and $\log \left(\int_{T} \exp (g(t)) \mathrm{d} \vartheta(t)\right) \leq a$ then

$$
\log \left(\int_{T} \exp \left(\frac{f(t)+g(t)}{2}\right) \mathrm{d} \vartheta(t)\right) \leq \log \left(\frac{1}{2} \exp (a)+\frac{1}{2} \exp (a)\right) \leq a .
$$

Therefore, $H_{f}=\log \left(\int_{T} \exp (f(t)) \mathrm{d} \vartheta(t)\right)$ is a convex function.

Proof of Lemma 3. By Taylor's expansion, the norm of the gradient can be defined as

$$
\left|\nabla H_{f_{N}}(x)\right|=\sup _{\left|v^{\prime}\right|=1} \lim _{\varepsilon \rightarrow 0+} \frac{H_{f_{N}}(x)-H_{f_{N}}\left(x+\varepsilon v^{\prime}\right)}{\varepsilon} .
$$

For each $b<a, H_{f_{N}}(x)=a$, and $x^{*}=\arg \inf _{z \in V_{N, b}}|x-z|$, let $v^{*}=\left(x^{*}-x\right) /\left|x^{*}-x\right|$. Then

$$
\left|\nabla H_{f_{N}}(x)\right| \geq \lim _{\varepsilon \rightarrow 0+} \frac{H_{f_{N}}(x)-H_{f_{N}}\left(x+\varepsilon v^{*}\right)}{\varepsilon} .
$$

By the convexity of $H$ (Lemma 2) and the fact that $f_{N}\left(x+\varepsilon v^{*}, \cdot\right)$ is a linear function of $\varepsilon$, $H_{f_{N}}\left(x+\varepsilon v^{*}\right)$ is a convex function of $\varepsilon$ and, therefore,

$$
\frac{H_{f_{N}}(x)-H_{f_{N}}\left(x+\varepsilon v^{*}\right)}{\varepsilon}
$$

is a positive and decreasing function of $\varepsilon$. We choose $\varepsilon=\left|x^{*}-x\right|=\rho\left(x, V_{N, b}\right)$. Then $\left|H_{f_{N}}(x)-H_{f_{N}}\left(x+\varepsilon v^{*}\right)\right|=a-b$. Thus, we obtain the bound

$$
\left|\nabla H_{f_{N}}(x)\right| \geq \frac{a-b}{\rho\left(x, V_{N, b}\right)} .
$$

As the proof of Lemma 4 requires Lemma 6, we prove Lemma 6 first.

Proof of Lemma 6. Lemma 10 (stated in Appendix B; see also [17] and Theorem 3.1 of [7]) implies that the following inequality holds:

$$
\int_{B^{c}} J(\rho(x, B)) \mathrm{d} \mu_{N}(x) \leq \int_{t_{B}}^{\infty} J\left(u-t_{B}\right) \mathrm{d} \Phi(u) .
$$

In view of this, we have

$$
\begin{aligned}
\int_{B_{r}^{c}} J(\rho(x, B)) \mathrm{d} \mu_{N}(x) \leq & \int_{B^{c}}[J(\rho(x, B)) I(\rho(x, B) \geq r)+J(r) I(\rho(x, B)<r)] \mathrm{d} \mu_{N}(x) \\
& -\int_{B_{r} \backslash B} J(r) I(\rho(x, B) \leq r) \mathrm{d} \mu_{N}(x) \\
\leq & \int_{t_{B}}^{\infty}\left[J\left(u-t_{B}\right) I\left(u-t_{B} \geq r\right)+J(r) I\left(u-t_{B}<r\right)\right] \mathrm{d} \Phi(u) \\
& -J(r)\left[\mu_{N}\left(B_{r}\right)-\mu_{N}(B)\right] .
\end{aligned}
$$

Note that

$$
1-\mu_{N}\left(B_{r}\right)=\int_{B^{c}} I(\rho(x, B) \geq r) \mathrm{d} \mu_{N}(x) \leq \int_{t_{B}}^{\infty} I\left(u-t_{B} \geq r\right) \mathrm{d} \Phi(u)=1-\Phi\left(t_{B}+r\right) .
$$


Then, $\mu_{N}\left(B_{r}\right) \geq \Phi\left(t_{B}+r\right)$ and further $\mu_{N}\left(B_{r}\right)-\mu_{N}(B) \geq \Phi\left(t_{B}+r\right)-\Phi\left(t_{B}\right)$. Substitute this result into (39) and note that

$$
\int_{t_{B}}^{\infty} J(r) I\left(u-t_{B}<r\right) \mathrm{d} \Phi(u)=J(r)\left[\Phi\left(t_{B}+r\right)-\Phi\left(t_{B}\right)\right] .
$$

We then obtain

$$
\int_{B_{r}^{c}} J(\rho(x, B)) \mathrm{d} \mu_{N}(x) \leq \int_{t_{B}+r}^{\infty} J\left(u-t_{B}\right) \mathrm{d} \Phi(u) .
$$

Proof of Lemma 4. Let

$$
a^{\prime}=\frac{a-b}{M a} b+b \quad \text { and } \quad V_{N, b}^{\prime}=\left\{z: H_{f_{N}}(z)<b \text { and } \sup _{t \in T}\left\{f_{N}(z, t)\right\}<M a\right\} .
$$

Thanks to the convexity of $V_{N, b}, \rho\left(x, V_{N, b}\right) \geq \rho\left(h(x), V_{N, b}\right)$ for all $x \in V_{N, a, 1}^{c}$. We want to apply Lemma 6 by considering $B=V_{N, b}$ and $V_{N, a, 1}^{c} \subset B_{\tau_{M, a, b}}^{c}$. Thus, we only need to show that, for each $x \in S_{a} \cap\left\{\sup \left\{\tilde{f}_{N}(x, t)\right\}<M a\right\}$,

$$
\rho\left(x, V_{N, b}\right) \geq \tau_{M, a, b}=\frac{a-b}{M a} \frac{a^{\prime}}{b} t_{N, b}^{\prime}
$$

By Lemma 3 and inequality (11), we have

$$
\rho\left(x, V_{N, b}\right) \geq(a-b) l_{x} \geq \frac{a-b}{M a} c_{x} .
$$

Therefore, we only need to show that

$$
c_{x} \geq \frac{a^{\prime}}{b} t_{N, b}^{\prime}
$$

For any $z \in V_{N, b}^{\prime}$,

$$
\begin{aligned}
\log \int_{T} \exp \left(\tilde{f}_{N}\left(z \frac{a^{\prime}}{b}, t\right)\right) \mathrm{d} t & =\log \int_{T} \exp \left(\tilde{f}_{N}\left(z+z \frac{a^{\prime}-b}{b}, t\right)\right) \mathrm{d} t \\
& \leq \log \int_{T} \exp \left(\tilde{f}_{N}(z, t)\right) \mathrm{d} t+\sup _{t \in T}\left\{\tilde{f}_{N}\left(z \frac{a^{\prime}-b}{b}, t\right)\right\} \\
& \leq b+\frac{a^{\prime}-b}{b} M a \\
& =a .
\end{aligned}
$$

Thus, we have $\mu_{N}\left(\left\{z a^{\prime} / b: z \in V_{N, b}^{\prime}\right\}\right) \leq F_{N}(a)$. Thanks to Lemma 11 (see Appendix B, and also Theorem 1 of [12]), we have

$$
\Phi\left(t_{N, b}^{\prime} \frac{a^{\prime}}{b}\right) \leq \mu_{N}\left(\left\{z \frac{a^{\prime}}{b}: z \in V_{N, b}^{\prime}\right\}\right) \leq F_{N}(a) .
$$

Consider an $x \in S_{a}$ and its unit vector $\boldsymbol{n}_{x}$ orthogonal to the tangent plane of $S_{a}$ at $\mathrm{x}$, denoted by $T_{x}$. According to the convexity of $V_{N, a}$, the entire set of $V_{N, a}$ lies on one side of $T_{x}$, which 
is the side opposite to $\boldsymbol{n}_{x}$. The above statement is equivalent to $V_{N, a} \subset\left\{z:\left\langle z, \boldsymbol{n}_{x}\right\rangle<c_{x}\right\}$, where $c_{x}=\left\langle x, \boldsymbol{n}_{x}\right\rangle$. Thus,

$$
F_{N}(a)=\mu_{N}\left(V_{N, a}\right) \leq \mu_{N}\left(\left\{z:\left\langle z, \boldsymbol{n}_{x}\right\rangle<c_{x}\right\}\right)=\Phi\left(c_{x}\right) .
$$

Combining the above inequality with (40), we obtain, for each $x \in S_{a} \cup V_{N, a, 1}^{c}, \Phi\left(t_{N, b}^{\prime} a^{\prime} / b\right) \leq$ $F_{N}(a) \leq \Phi\left(c_{x}\right)$ and, thus, $c_{x} \geq t_{b}^{\prime} a^{\prime} / b$. Therefore,

$$
\rho\left(x, V_{N, b}\right) \geq(a-b) l_{x} \geq \frac{a-b}{M a} c_{x} \geq \frac{a-b}{M a} \frac{a^{\prime}}{b} t_{N, b}^{\prime},
$$

which completes the proof of Lemma 6.

The next lemma provides a bound for the second derivative of $F(a)$ :

$$
D^{+} F_{N}^{\prime}(a):=\limsup _{\varepsilon \rightarrow 0} \frac{F_{N}^{\prime}(a+\varepsilon)-F_{N}^{\prime}(a)}{\varepsilon} .
$$

Lemma 8. Consider the probability space $\left(\mathbb{R}^{N}, \mathcal{B}\left(\mathbb{R}^{N}\right), \mu_{N}\right)$. Under the conditions of Theorem 1, when $G_{N}(a)>\frac{1}{2}$, for $\tilde{b}<a$ and $b<a$ such that $G_{N}(b)>\frac{1}{2}$, we have

$$
\begin{aligned}
D^{+} F_{N}^{\prime}(a) \leq \min _{M \geq 1}\left\{\left(1-\Phi\left(\tau_{M, a, b}+t_{N, b}\right)\right) C_{3}\left(a, b, \tau_{M, a, b}\right)\right. \\
\left.+\left(1-\Phi\left(r_{M, a, \tilde{b}}+t_{W_{N, \tilde{b}}}\right)\right) C_{4}\left(a, \tilde{b}, r_{M, a, \tilde{b}}\right)\right\},
\end{aligned}
$$

and when $G_{N}(a) \leq \frac{1}{2}$, we have

$$
D^{+} F_{N}^{\prime}(a) \leq\left(1-\Phi\left(r_{a, \tilde{b}}+t_{W_{N, \tilde{b}}}\right)\right) C_{4}\left(a, \tilde{b}, r_{a, \tilde{b}}\right),
$$

where $t_{W_{N, b}}=\Phi^{-1}\left(G_{N}(b)\right), r_{M, a, \tilde{b}}=(M a-\tilde{b}) / \sigma, r_{a, \tilde{b}}=(a-\tilde{b}) / \sigma$,

$$
\begin{aligned}
\tau_{M, a, b}= & \frac{a-b}{M a}\left(\frac{a-b}{M a}+1\right) t_{N, b}^{\prime}, \\
C_{3}\left(a, b, \tau_{M, a, b}\right)= & \frac{1}{(a-b)^{2}}\left(\frac{10}{3} \sum_{i=0}^{2} \frac{\tau_{M, a, b}^{2} 2 !}{(2-i) ! \tau_{M, a, b}^{i}\left(t_{N, b}+\tau_{M, a, b}\right)^{i}}\right. \\
& \left.+\frac{a^{2} M^{2}}{(a-b)^{2}} \sum_{i=0}^{4} \frac{\tau_{M, a, b}^{4} 4 !}{(4-i) ! \tau_{M, a, b}^{i}\left(t_{N, b}+\tau_{M, a, b}\right)^{i}}\right), \\
C_{4}\left(a, \tilde{b}, r_{M, a, \tilde{b}}\right)= & \frac{10}{3(a-\tilde{b})^{2}} \sum_{i=0}^{2} \frac{r_{M, a, \tilde{b}}^{2} 2 !}{(2-i) ! r_{M, a, \tilde{b}}^{i}\left(t_{W_{N, \tilde{b}}}+r_{M, a, \tilde{b}}\right)^{i}} \\
& +\frac{r^{4}}{(a-\tilde{b})^{4}} \sum_{i=0}^{4} \frac{4, a, \tilde{b}}{(4-i) ! r_{M, a, \tilde{b}}^{i}\left(t_{W_{N, \tilde{b}}}+r_{M, a, \tilde{b}}\right)^{i}} \\
& +\frac{2 \sigma \tilde{b}}{(a-\tilde{b})^{4}} \sum_{i=0}^{5} \frac{r_{M, a, b}^{5} 5 !}{(5-i) ! r_{M, a, \tilde{b}}^{i}\left(t_{W_{N, \tilde{b}}}+r_{M, a, \tilde{b}}\right)^{i}} \\
& +\frac{\sigma^{2}}{(a-\tilde{b})^{4}} \sum_{i=0}^{6} \frac{r_{M, a, \tilde{b}}^{6} 6 !}{(6-i) ! r_{M, a, \tilde{b}}^{i}\left(t_{W_{N, \tilde{b}}}+r_{M, a, \tilde{b}}\right)^{i}} .
\end{aligned}
$$


Proof. As in step 1 in Section 3.1.1, we have a volume integral bound for $F_{N}^{\prime}(a)$ :

$$
D^{+} F_{N}^{\prime}(a) \leq \int_{V_{N, a}^{c}} l_{h(x)}^{2}\left(\left(c_{x}^{+}\right)^{2}+\frac{10}{3}\right) \mathrm{d} \mu_{N}(x) .
$$

The proof the above bound follows an argument in [20] (in particular, pages 850-851 therein) and is therefore omitted.

Similarly to the proof in Section 3, consider the partition of $V_{N, a}^{c}: V_{N, a}^{c}=V_{N, a, 1}^{c} \cup V_{N, a, 2}^{c}$ for $M \geq 1$. We have

$$
D^{+} F_{N}^{\prime}(a) \leq \int_{V_{N, a, 1}^{c}} l_{h(x)}^{2}\left(\left(c_{x}^{+}\right)^{2}+\frac{10}{3}\right) \mathrm{d} \mu_{N}(x)+\int_{V_{N, a, 2}^{c}} l_{h(x)}^{2}\left(\left(c_{x}^{+}\right)^{2}+\frac{10}{3}\right) \mathrm{d} \mu_{N}(x) .
$$

Similarly as in the above derivation for (14), we have, for $a$ and $b$ such that $G_{N}(a)>G_{N}(b)>$ $\frac{1}{2}$,

$$
\begin{aligned}
\int_{V_{N, a, 1}^{c}} & l_{h(x)}^{2}\left(\left(c_{x}^{+}\right)^{2}+\frac{10}{3}\right) \mathrm{d} \mu_{N}(x) \\
\leq & \int_{V_{N, a, 1}^{c}} \frac{\rho\left(h(x), V_{N, b}\right)^{2}}{(a-b)^{2}}\left(\frac{\rho\left(h(x), V_{N, b}\right)^{2}}{(a-b)^{2}}\left(\sup _{t \in T}\left\{f_{N}(h(x), t)\right\}\right)^{2}+\frac{10}{3}\right) \mathrm{d} \mu_{N}(x) \\
\leq & \int_{\tau_{M, a, b}+t_{N, b}}^{\infty} \frac{\left(u-t_{N, b}\right)^{2}}{(a-b)^{2}}\left(\frac{\left(u-t_{N, b}\right)^{2}}{(a-b)^{2}}(M a)^{2}+\frac{10}{3}\right) \mathrm{d} \Phi(u) \\
= & \left(1-\Phi\left(\tau_{M, a, b}+t_{N, b}\right)\right) C_{3}\left(a, b, r_{M, a, b}\right) .
\end{aligned}
$$

Similarly as in the above derivation of (19), we have, for $\tilde{b}<a$,

$$
\begin{aligned}
\int_{V_{N, a, 2}^{c}} & l_{h(x)}^{2}\left(\left(c_{x}^{+}\right)^{2}+\frac{10}{3}\right) \mathrm{d} \mu_{N}(x) \\
\leq & \int_{V_{N, a, 2}^{c}} \frac{\rho\left(h(x), V_{N, \tilde{b}}\right)^{2}}{(a-\tilde{b})^{2}}\left(\frac{\rho\left(h(x), V_{N, \tilde{b}}\right)^{2}}{(a-\tilde{b})^{2}}\left(\sup _{t \in T}\left\{f_{N}(h(x), t)\right\}\right)^{2}+\frac{10}{3}\right) \mathrm{d} \mu_{N}(x) \\
\leq & \int_{r_{M, a, \tilde{b}}+t_{W_{N, \tilde{b}}}}^{\infty} \frac{\left(u-t_{W_{N, \tilde{b}}}\right)^{2}}{(a-\tilde{b})^{2}}\left(\frac{\left(u-t_{W_{N, \tilde{b}}}\right)^{2}}{(a-\tilde{b})^{2}}\left(\left(u-t_{W_{N, \tilde{b}}}\right) \sigma+\tilde{b}\right)^{2}+\frac{10}{3}\right) \mathrm{d} \Phi(u) \\
\leq & \left(1-\Phi\left(r_{M, a, \tilde{b}}+t_{W_{N, \tilde{b}}}\right)\right) C_{4}\left(a, \tilde{b}, r_{M, a, \tilde{b}}\right),
\end{aligned}
$$

where $t_{W_{N, \tilde{b}}}=\Phi^{-1}\left(G_{N}(\tilde{b})\right), r_{M, a, \tilde{b}}=(M a-\tilde{b}) / \sigma$, and the last inequality follows from Lemma 5. Note that, when $G_{N}(a)<\frac{1}{2}$, we take $M=1$ and our conclusion holds.

\section{Appendix B. Supplementary material}

We present the Borel-TIS lemma that is proved by [7] and [21].

Lemma 9. (Borel-TIS.) Let $f(t), t \in U(U$ is a parameter set), be a mean zero Gaussian random field; $f$ is almost surely bounded on $\mathcal{U}$. Then

$$
\mathbb{E}\left(\sup _{u} f(t)\right)<\infty
$$


and

$$
\mathbb{P}\left(\max _{t \in \mathcal{U}} f(t)-\mathbb{E}\left(\max _{t \in \mathcal{U}} f(t)\right) \geq b\right) \leq \mathrm{e}^{-b^{2} / 2 \sigma_{u}^{2}},
$$

where

$$
\sigma_{u}^{2}=\max _{t \in \mathcal{U}} \operatorname{var}(f(t))
$$

The following well-known isoperimetric inequality is due independently to Borell [7] and Sudakov and Tsirel'son [17].

Lemma 10. Let $B$ be a measurable set of positive measure in $\mathbb{R}^{N}$, and let

$$
\mu_{N}(B)=\Phi(a)
$$

Then we have, for every $r \geq 0$,

$$
\mu_{N}\left(B_{r}\right) \geq \Phi(a+r),
$$

where $B_{r}=B+r U=\{x+r y: x \in B, y \in U\}$ and $U$ is the unit ball in $\mathbb{R}^{N}$.

The following result follows from Theorem 1 of [12].

Lemma 11. For any convex set $B$ in $\mathbb{R}^{n}$ and half-space $H=\left\{x \in \mathbb{R}^{N}:\langle x, \boldsymbol{n}\rangle \leq a\right\}$ with some real number a and some unit vector $\boldsymbol{n}$ such that

$$
\mu_{N}(B) \geq \mu_{N}(H)=\Phi(a),
$$

we have, for every $r \geq 1$,

$$
\mu_{N}(r B) \geq \mu_{N}(r H)=\Phi(r a)
$$

where $B=\{r x: x \in B\}$.

\section{Acknowledgement}

This research was supported in part by the Institute of Education Sciences, through grant numbers R305D100017, NSF CMMI-1069064, and NSF SES-1123698.

\section{References}

[1] Adler, R. J. and Taylor, J. E. (2007). Random Fields and Geometry. Springer, New York.

[2] Adler, R. J., Blanchet, J. H. and LiU, J. (2012). Efficient Monte Carlo for high excursions of Gaussian random fields. Ann. Appl. Prob. 22, 1167-1214.

[3] Asmussen, S. and Rojas-Nandayapa, L. (2008). Asymptotics of sums of lognormal random variables with Gaussian copula. Statist. Prob. Lett. 78, 2709-2714.

[4] Azais, J.-M. And Wschebor, M. (2008). A general expression for the distribution of the maximum of a Gaussian field and the approximation of the tail. Stoch. Process. Appl. 118, 1190-1218.

[5] Blanchet, J., Juneja, J. and Rojas-Nandayapa, L. (2013). Efficient tail estimation for sums of correlated lognormals. To appear in Ann. Operat. Res.

[6] Bogachev, V. I. (1998). Gaussian Measures (Math. Surveys Monogr. 62). American Mathematical Society, Providence, RI.

[7] Borell, C. (1975). The Brunn-Minkowski inequality in Gauss space. Invent. Math. 30, 207-216.

[8] Davis, R. A., Dunsmuir, W. T. M. And Wang, Y. (2000). On autocorrelation in a Poisson regression model. Biometrika 87, 491-505.

[9] Duffie, D. And Pan, J. (1997). An overview of value at risk. J. Derivatives 4, 7-49.

[10] Dufresne, D. (2001). The integral of geometric Brownian motion. Adv. Appl. Prob. 33, 223-241.

[11] Foss, S. AND Richards, A. (2010). On sums of conditionally independent subexponential random variables. Math. Operat. Res. 35, 102-119.

[12] Landau, H. J. And Shepp, L. A. (1970). On the supremum of a Gaussian process. Sankhyā A 32, 369-378. 
[13] LiU, J. (2012). Tail approximations of integrals of Gaussian random fields. Ann. Prob. 40, 1069-1104.

[14] LiU, J. AND XU, G. (2012). Some asymptotic results of Gaussian random fields with varying mean functions and the associated processes. Ann. Statist. 40, 262-293.

[15] Mitra, A. AND Resnick, S. I. (2009). Aggregation of rapidly varying risks and asymptotic independence. $A d v$. Appl. Prob. 41, 797-828.

[16] Piterbarg, V. I. (1996). Asymptotic Methods in the Theory of Gaussian Processes and Fields. American Mathematical Society, Providence, RI.

[17] Sudakov, V. N. AND Tsirel'son, B. S. (1974). Extremal properties of half-spaces for spherically invariant measures. Zap. Nauchn. Sem. LOMI 41, 14-24.

[18] Sun, J. Y. (1993). Tail probabilities of the maxima of Gaussian random fields. Ann. Prob. 21, 34-71.

[19] Taylor, J. E. ANd Adler, R. J. (2003). Euler characteristics for Gaussian fields on manifolds. Ann. Prob. 31, 533-563.

[20] Tsirel'son, V. S. (1975). The density of the distribution of the maximum of a Gaussian process. Theory Prob. Appl. 20, 847-856.

[21] Tsirel'son, B. S., Ibragimov, I. A. and Sudakov, V. N. (1976). Norms of Gaussian sample functions. In Proc. 3rd Japan-USSR Symp. on Probability Theory (Tashkent, 1975; Lecture Notes Math. 550), Springer, Berlin, pp. 20-41.

[22] Yor, M. (1992). On some exponential functionals of Brownian motion. Adv. Appl. Prob. 24, 509-531. 